\title{
Overhearing-Assisted Optimization of Address Autoconfiguration in Position-Aware VANETs
}

\author{
Marco Gramaglia, Ignacio Soto, Carlos J. Bernardos, and Maria Calderon
}

\begin{abstract}
Vehicular networks allow vehicles to exchange information that can be used to improve traffic efficiency and drivers' safety. In addition to these new applications, Internet connectivity is also expected to be available in the cars of the near future, speeding up the global adoption of vehicular communication systems. One of the requirements for connecting vehicles to the Internet is their ability to autoconfigure Internet Protocol (IP) addresses. In this paper, we propose an optimization to Geographically Scoped stateless Address Configuration (GeoSAC), which is an IP address autoconfiguration mechanism for geographically aware location vehicles. The benefits of this optimization are twofold: 1) It can reduce the IP address configuration time, and 2) it can be used to reduce the signaling overhead of GeoSAC. The optimization requires no changes to the GeoSAC operation, being fully compatible with the original solution. We derive an analytical model for the probability of our optimization being effective in realistic scenarios and for the IP address configuration time. We also provide a thorough evaluation of the performance improvements of the optimization, including simulations with a realistic model for wireless technology, real vehicular traces, and experiments with a real prototype, which provide strong support for our analytical model.
\end{abstract}

Index Terms-Geographically Scoped stateless Address Configuration (GeoSAC), geonetworking, Internet Protocol (IP) address autoconfiguration, vehicular ad hoc networks (VANETs).

\section{INTRODUCTION}

$\mathbf{V}$ EHICULAR communications are going to have an impact on the way we drive in the not-too-distant future. Driven by the goals of improving road safety and efficiency, governments, car manufacturers, and telecommunication players are working toward the definition of a communications architecture that enables vehicles to benefit from communication capabilities. Among the different candidate architectures that could be applied to tackle the problem of vehicular communications, vehicular ad hoc networks (VANETs) have been adopted by the majority of the existing initiatives due to their

Manuscript received August 6, 2010; revised December 20, 2010 and March 29, 2011; accepted May 16, 2011. Date of publication June 2, 2011; date of current version September 19, 2011. This work was supported by the Spanish Ministry for Science and Innovation through the I-MOVING Project TEC2010-18907. The review of this paper was coordinated by Prof. N. Kato.

M. Gramaglia is with the Department of Telematics Engineering, Universidad Carlos III de Madrid, 28911 Leganes, Spain, and also with the Institute IMDEA Networks, 28918 Leganes, Spain (e-mail: marco.gramaglia@ imdea.org).

I. Soto is with the Department of Telematics Engineering, Universidad Politécnica de Madrid, 28040 Madrid, Spain (e-mail: isoto@dit.upm.es).

C. J. Bernardos and M. Calderon are with the Department of Telematics Engineering, Universidad Carlos III de Madrid, 28911 Leganes, Spain (e-mail: cjbc@it.uc3m.es; maria@it.uc3m.es).

Color versions of one or more of the figures in this paper are available online at http://ieeexplore.iee.org.

Digital Object Identifier 10.1109/TVT.2011.2158597 decentralized nature, which supports an unmanaged operation without infrastructure involvement. The primary advantage of deploying this kind of self-organized network is that timely critical applications, such as life-safety applications, can be implemented by letting vehicles directly communicate with each other instead of relying on a centralized entity. Although safety-related applications have been the main focus of research and standardization efforts so far, ensuring coexistence with other types of applications, specifically nonsafety-related applications (e.g., infotainment or generic Internet applications), has also been considered. Integrating the Internet Protocol (IP) into the system architecture not only enables vehicles to access classical and new Internet applications but takes advantage of all the operational experience with IP networks and the huge amount of implementations and resources that are available as well. This will help speed up the deployment of vehicular communication systems because the manufacturers and operators involved can save money by reusing existing resources, and users will see the additional benefit of the installation of a communication system in their cars (i.e., it would be not only safety oriented but also allow for Internet-based services).

The feasibility of Internet access from vehicles has been addressed in several research studies, assuming single-hop access in some cases [1], [2] and multihop access in others [3], [4] to the Internet; they have concluded that the use of IP-based applications over VANETs is viable. However, the provision of vehicles with Internet access by partially reusing the multihop VANET system architecture that is used for supporting safety applications is a problem that has yet to be extensively researched [5]-[7]. Note that we highlight the multihop VANET nature of the scenario because enabling Internet connectivity by using a single-hop access technology of wide-area coverage, such as Third-Generation (3G), poses no significant design challenge, although it has cost and performance drawbacks. Some functionalities will be required to bring IP into multihop vehicular networks:

1) the capability of vehicles to autoconfigure an IP address;

2) IP mobility mechanisms suited for the multihop vehicular scenario;

3) mechanisms for an efficient transmission and forwarding of IP datagrams within the VANET.

To tackle the address autoconfiguration problem, we proposed a mechanism in [7] called Geographically Scoped stateless Address Configuration (GeoSAC) that adapts the existing IPv6 StateLess Address AutoConfiguration (SLAAC) [8], [9] mechanisms to work in position-aware VANETs. In this type of VANET, nodes know their geographical position (e.g., by 
using a GPS receiver) and use geographical routing algorithms to forward data. This approach has been adopted by major consortia and standardization committees, such as the Car-to-Car Communications Consortium ${ }^{1}$ (C2C-CC) and the European Telecommunications Standards Institute Technical Committee Intelligent Transport Systems (ETSI TC ITS ${ }^{2}$ ). The ETSI TC ITS system architecture, the basic operation of GeoSAC, and the motivations supporting the need for GeoSAC optimization are presented in Section II.

In this paper, we propose an optimization mechanism for GeoSAC based on nodes overhearing (when possible) information about the IP prefixes that they might use to configure an IP address in the future (due to the movement of the nodes). This method saves time that would otherwise be needed to reactively determine the preceding information. The overhearing-assisted optimization mechanism and its probability of being effectively used are described in Section III. We mathematically analyzed the optimization and compared it with the performance of the original GeoSAC solution in Section IV. The improvements in terms of address configuration time reduction and signaling saving and their impact when combined with the use of an IP mobility solution were experimentally evaluated via simulations in different scenarios. Vehicular traces obtained from highways in Spain were also used to validate our analysis. Finally, we present in Section V the results of an experimental evaluation conducted with a real prototype in a testbed composed of 40 nodes. The obtained results show that our optimization mechanism is feasible and could be used to significantly improve the performance of GeoSAC. We conclude this paper in Section VI.

\section{BACKGROUND AND RATIONALE}

\section{A. Related Work}

The multihop nature of VANETs and their lack of a single multicast-capable link for signaling prevent current IPv6 address autoconfiguration related protocol specifications from being used as-is in VANETs. This fact applies both to the IPv6 SLAAC [8], [9] and to its stateful counterpart dynamic host configuration protocol (DHCPv6) [10]. In addition, due to node mobility, a vehicular network may get partitioned or independent networks may merge, causing additional problems that need to be tackled [11].

There are several studies in the literature that propose to adapt DHCP mechanisms to work on VANET environments following distributed [12] or centralized [13] approaches. The vehicular address configuration protocol [12] uses a distributed DHCP service. It consists of a dynamic leader-based approach in which addresses are assigned by dynamically elected leaders running a DHCP server. Leaders coordinate with other within a certain distance (i.e., within the SCOPE parameter) to maintain updated information on currently assigned addresses and avoid the existence of duplicates. By contrast, the centralized address configuration (CAC) [13] employs a centralized DHCP

\footnotetext{
${ }^{1}$ http://www.car-to-car.org/

${ }^{2} \mathrm{http} / / /$ portal.etsi.org/its/its_tor.asp
}

server located in the infrastructure that can provide unique IP addresses to all the vehicles in an urban area. In this case, access routers for the Internet relay configure messages between vehicles and the central DHCP server, which can ensure that vehicles will not be configured with duplicate IP addresses. The main weakness of DHCP-based proposals is the time required to acquire or lease an IP address [1].

Enabling address autoconfiguration by adapting the IPv6 SLAAC mechanisms to work in position-aware multihop VANETs has also been researched [6], [7]. In the work of Choi et al. [6], each vehicle obtains a different IPv6 prefix [by means of a router advertisement (RA) message] from the access router, and all the IP signaling messages are exchanged through a virtual point-to-point link set between the vehicle and the access router. In the case of GeoSAC [7], the concept of an IPv6 link is extended to a specific geographic area associated with a point of attachment (e.g., an access router), and all the vehicles within the area share the same IPv6 prefix.

The main drawback of the work of Choi et al. is the need for a proactive mechanism to discover the access router to the Internet. This mechanism is needed both initially and after movements, but this paper does not tackle the issue of how mobility across multiple access routers is to be handled. As detailed in the next section, however, GeoSAC (thanks to the concept of an IPv6 link covering a specific geographic area associated with an access router) enables the standard IPv6 mechanisms of movement detection to be used as is.

\section{B. GeoSAC and the ETSI TC ITS System Architecture}

GeoSAC [7] is a mechanism for the application of an IPv6 automatic address configuration technique to vehicular networks that is based on combining standardized IPv6 schemes with geographical routing functionalities. GeoSAC is defined in [7] for the VANET system architecture proposed by the C2C-CC. The ETSI TC ITS is defining a reference system [14] that is based on the $\mathrm{C} 2 \mathrm{C}-\mathrm{CC}$ recommendations. GeoSAC and the optimization proposed in this paper work in both communication architectures because they are basically the same. The ETSI TC ITS is the technical committee that has received a standardization mandate from the European Commission for the development of short-range ITS communication protocols. We next describe the ETSI TC ITS reference system and assume its use in the rest of this paper.

The ETSI TC ITS is currently developing a set of protocols and algorithms that define a harmonized communication system for European ITS applications, taking into account industry requirements, particularly those coming from the $\mathrm{C} 2 \mathrm{C}-\mathrm{CC}$. In the ETSI TC ITS network architecture [14], vehicles are equipped with devices called communication and control units (CCUs), which implement the ETSI protocol stack (see Figs. 1 and 2). Vehicles can communicate with each other or with fixed roadside ITS stations (also called roadside units: RSUs) installed along roads. CCUs and RSUs implement the same network layer functionalities and form a self-organizing network. The RSUs can be connected to a network infrastructure: most likely an IP-based network. Onboard application hosts, including passenger devices attached to the vehicle onboard 


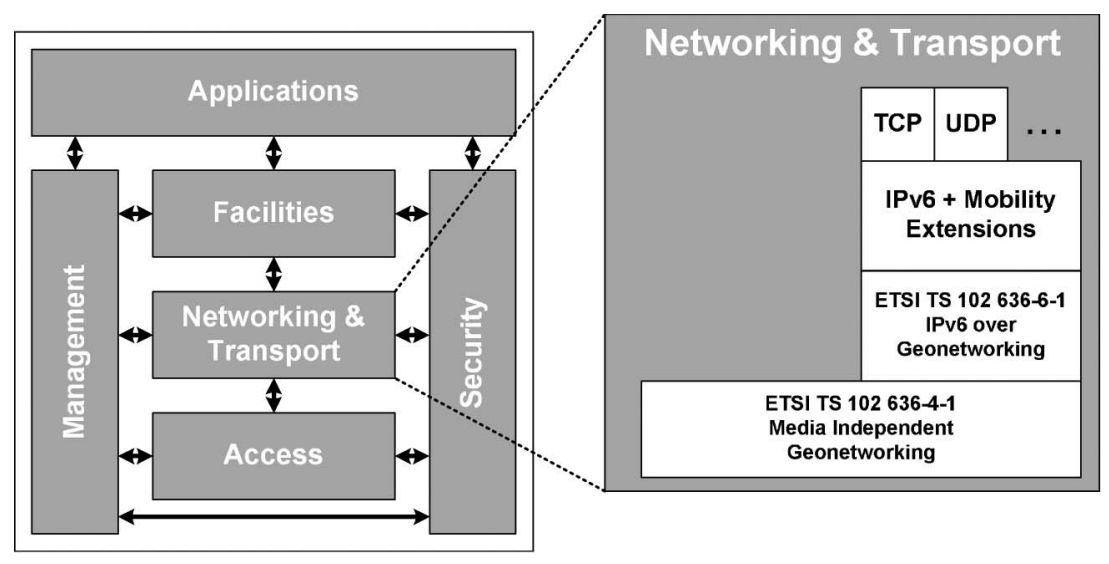

Fig. 1. ETSI TC ITS protocol architecture.

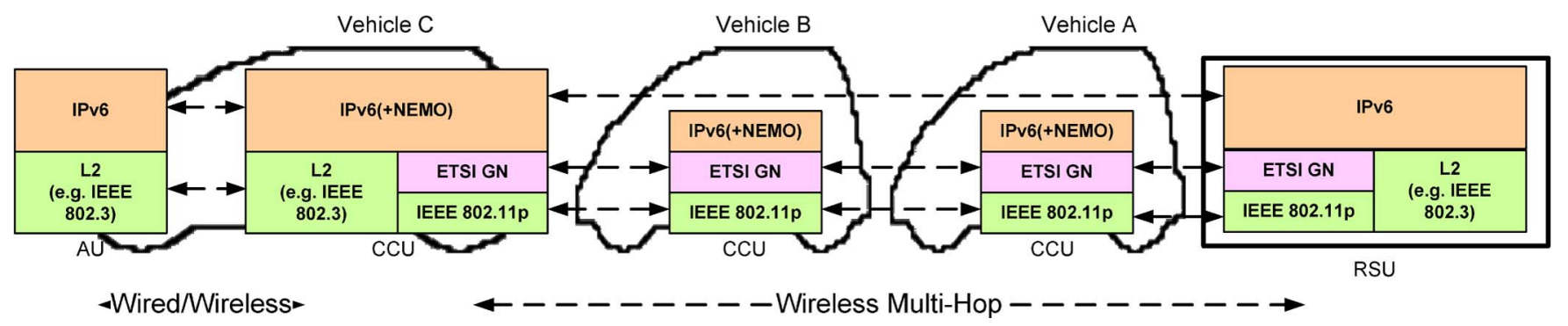

Fig. 2. GeoSAC forwarding within an area and affected protocol layers.

system, are called application units (AUs). Passenger devices are assumed to have a standard IPv6 protocol stack, whereas CCUs act as gateways for the in-vehicle network and are optionally enhanced with the NEtwork MObility (NEMO) basic support protocol [15].

The ETSI GeoNetworking (GN) protocol [16] plays the role of a network layer protocol in the ETSI TC ITS architecture, providing routing and addressing facilities in the upper layers. Given the nature of vehicular communications, a geographicalbased routing and addressing approach has been adopted. An intermediate node forwards a packet to the direct neighbor that is closest to the geographic position of the destination, which is an operation known as greedy forwarding. Therefore, each node must be aware of the position of its direct neighbors and the position of the final destination. To this end, nodes send periodic beacon messages informing neighboring nodes about their identifier, position, and other relevant information. As for the position of the final destination, this information is provided by a location service. This functionality may be centralized (i.e., nodes updating their new locations on a location server) or distributed (e.g., the source node floods a message asking for the position of the destination node). Each node has one or more identifiers at the GN layer that are resolved to the position of the node by the location service.

GeoSAC [7] adapts the existing IPv6 SLAAC [8], [9] mechanisms to geographic addressing and networking by extending the concept of an IPv6 link to a specific geographical area associated with a point of attachment. In GeoSAC, the ETSI GN layer [16] plays the role of sub-IP layer (see Fig. 1), dealing with ad hoc routing by using geographic location information and presenting to the IPv6 layer a flat network topology. Conse- quently, the link seen by the IPv6 layer includes nodes that are not directly reachable but are portrayed as such by the sub-IP layer (see Fig. 3). This layer provides IPv6 with a multicast link that includes a nonoverlapping partition of the VANET formed by all the nodes within a certain geographical area [17]. The ETSI GN layer also avoids layers above to deal with the complexity given by using geographical information for routing and broadcasting. The ETSI GN layer geobroadcasting is used by GeoSAC to shape multicast/broadcast messages to a geographical area.

The RSU sends out standard IPv6 RA messages ${ }^{3}$ that reach the nodes currently located within a well-defined area, and the nodes can then generate IPv6 addresses by appending their network identifier (derived from the identifier used at the GN layer) to the received IPv6 prefix. RA multicasting is scoped geographically; therefore, at the GN layer, a vehicle only forwards an RA if the RSU that generated it is placed within the same GeoSAC area as the receiving node. We use the example shown in Fig. 3 to explain the way GeoSAC makes use of the ETSI GN layer to logically deliver packets between two nodes that, although communicating via multiple wireless hops, are logically attached to the same logical IP link. Suppose a device within Vehicle $\mathrm{C}$ wants to communicate with a node on the Internet. At the IP layer, Vehicle $\mathrm{C}$ and the RSU are directly connected (i.e., one hop distance), and therefore, Vehicle C uses the RSU as the

\footnotetext{
${ }^{3}$ Note that the GeoSAC solution could be applied to multiple RSUs acting as bridges connected to one single access router (which sends RAs). This solution may be a good deployment choice in scenarios where single-hop connectivity to the infrastructure is preferred, and reducing the number of IPv6 address changes is also required (e.g., city environment).
} 


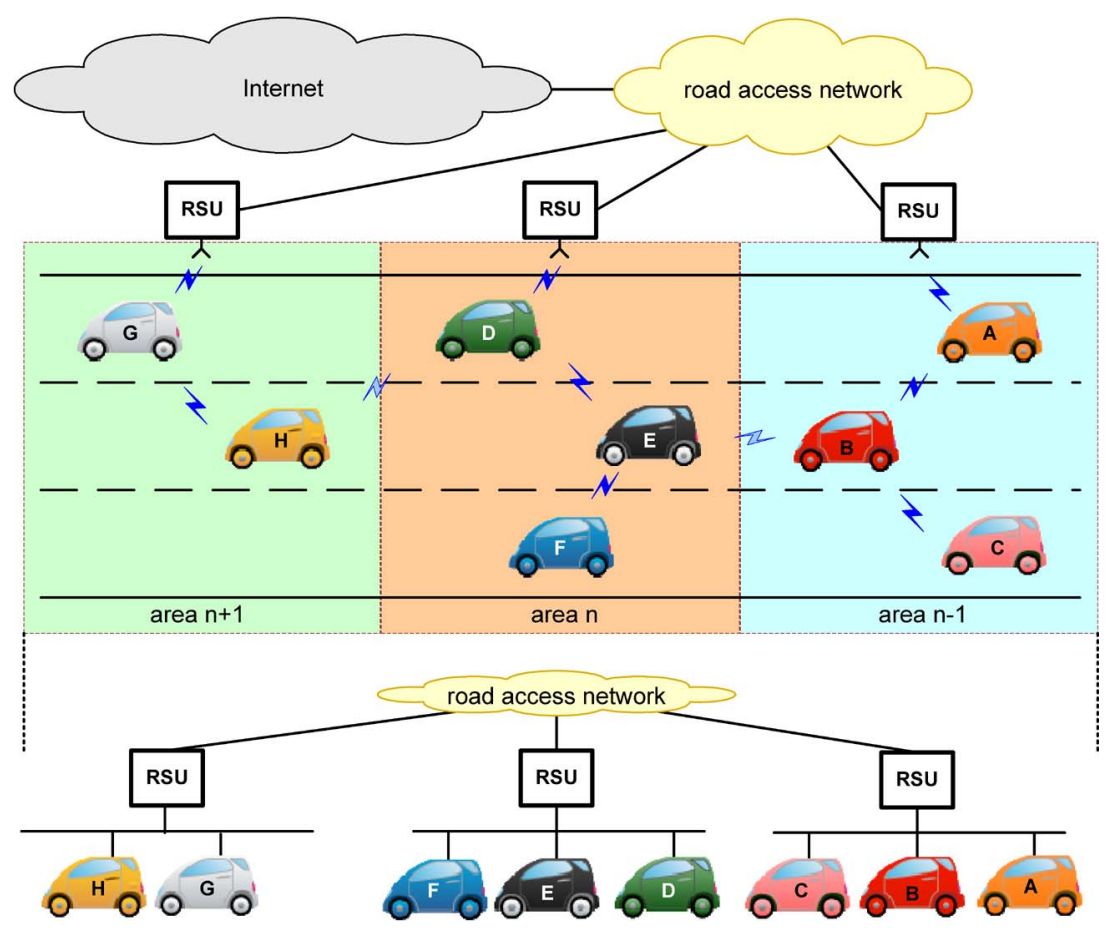

Fig. 3. ETSI TC ITS system architecture and GeoSAC area partitioning.

IP next-hop for the packets that it sends to the Internet. For that single-hop IP data forwarding to happen, Vehicle C must send the packets to Vehicle B, which forwards them to Vehicle A, which finally delivers them to the RSU. Note that this multihop forwarding is required because Vehicle $\mathrm{C}$ is not within the radio coverage (i.e., a single hop) of the RSU. If NEMO support is enabled, then no changes are required in the operation of the mobility protocol, as the multihop nature of the vehicular network is hidden from the IP stack, due to the use of GeoSAC and the ETSI TC ITS GN protocol. It is also worth mentioning that vehicles learn the geographic position of the RSUs from the RAs that they broadcast because this information is included in the GN protocol header of the frames.

The previous example shows that, in a system architecture based on short-range communication devices, the effective provisioning of Internet-based applications over multihop communication strongly depends on mobility. Single-hop vehicular Internet access based on wireless LAN (WLAN) has already been investigated in highway scenarios [18], for which it was concluded that the link between the CCU and the RSU is stable enough to allow for several types of applications.

\section{Reducing the IP Address Autoconfiguration Time and Signaling Overhead}

This section summarizes the main reasons why optimizing the mechanism used to provide nodes with an IPv6 address is important in the context of vehicular communications. The benefits from this optimization are twofold: 1) reducing the IP address configuration time and 2) minimizing the signaling overhead caused by the solution.

Because the concept of an IPv6 link is associated with a specific geographical area, i.e., in GeoSAC, each vehicle must stop using its old IP address and configure a new one every time it changes areas. This reconfiguration involves a time during which the vehicle cannot communicate, lasting until a valid IP address is configured and becomes usable. We call this time configuration time $T_{\text {conf }}$, and it is formally defined as the time elapsed from the moment a vehicle enters a new geographical area (thereby losing the connectivity to the old RSU) until the moment it starts using the newly configured global IPv6 address. Obviously, the shorter the time required to configure a new address, the better because the interruption time will be shorter. Unless additional mechanisms are in place, each time a vehicle changes its IP address, it must restart all the existing communications. IP mobility solutions (e.g., Mobile IPv6 [19]) have been designed to enable IP address changing without breaking ongoing communications. To provide mobility support to the network formed by the CCU and the AUs, NEMO solutions have been defined [15] and further refined for the vehicular scenario [20]. The use of IP mobility protocols solves the problem of communication disruptions due to the change of IP address, but it does not avoid the interruption time caused each time the vehicle changes its IP address. This time is typically increased when IP mobility solutions are used because of the additional time required to complete the signaling with the mobility anchor point [e.g., the home agent (HA) in mobile IPv6/NEMO]. It should also be noted that due to the high mobility of nodes, it is even more important to reduce the overall interruption time (which is called handover latency) because handovers are more frequent than in other scenarios [21].

There is another metric worth improving, i.e., the signaling overhead. To configure a new address in GeoSAC, a vehicle waits to receive an unsolicited RA from the RSU of the new area; therefore, the configuration time is bound to the frequency with which the RSU of the area is sending RAs. In addition, 
due to the way the ETSI GN and GeoSAC abstract an IPv6 link (mapping it to a geographical area), each RA multicast by an RSU is actually flooded within the area managed by the RSU, which therefore occupies the wireless media for more time than does one single-frame transmission. Therefore, reducing the frequency of unsolicited RAs is even more important than in a nonvehicular single-hop wireless scenario.

Driven by these two goals, reducing the address configuration time and keeping the signaling overhead to a minimum (note that there is a tradeoff here), we next propose an overhearing-assisted optimization mechanism for GeoSAC.

\section{OVERHEARING-ASSISTED OPTIMIZATION FOR GEOSAC}

In this section, we present an extension to the original GeoSAC that provides an important performance improvement and a signaling overhead reduction. An overview of the mechanism is first provided, and then the probability of achieving seamless IP address reconfiguration is analytically modeled.

\section{A. Solution Overview}

Our approach aims at reducing the IP address configuration time due to physical node movements that lead to a change of geographical area of the IPv6 link and ultimately of the IPv6 address. While the node is configuring a new IPv6 address (configuration time), the vehicle cannot communicate and has to defer its ongoing communications until a new and valid IP address is configured and becomes usable. Note that even if perfect juxtaposition of areas can logically be obtained in GeoSAC, in a practical scenario, this separation does not really exist at the physical layer. Nodes within radio range of the forwarder of an RA located in an adjacent area also receive the RA. The original GeoSAC mechanism [7] mandates that this RA should be filtered out at the ETSI GN layer to achieve perfect logical area division. However, nodes can benefit from overhearing RAs generated at areas other than the one where the receiving node is located. This way, vehicles would be able to learn the IPv6 prefix used in a neighboring area before actually entering it, and they would be able to precompute the IPv6 address and default router configuration that should be used when located in that area (i.e., just after crossing the area border).

When a vehicle overhears RAs from multiple neighboring areas (e.g., cities, road intersections, etc.), it stores the overheard RAs for some time. The vehicle learns the geographical area from which the RA was sent by using the destination information at the ETSI GN layer, which is set to the geographical area by the RSU when multicasting RAs. By storing these RA-area pairs, the vehicle will be able to configure an IP address without waiting for an unsolicited RA if it later enters one of the areas about which it has knowledge.

This overhearing-assisted optimization allows shortening of the average IP address configuration time in addition to a potential reduction of the required overhead. By enabling this optimization, GeoSAC improves its performance because nodes that successfully overhear an RA from an area where they later enter can start using their new addresses without waiting for a

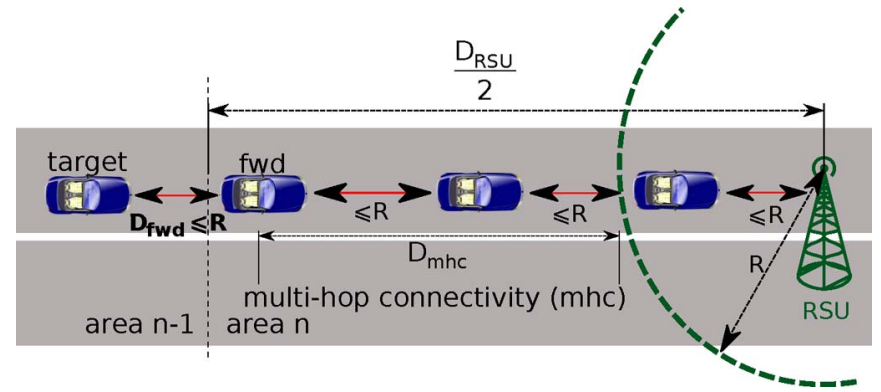

Fig. 4. Overhearing-assisted optimization overview and terminology.

new RA and with no extra overhead. The improvement obtained depends on the mobility conditions inside the VANET and does not limit the benefits achieved from using nonoverlapping areas that are described in [7].

This optimization has a cost in terms of additional complexity at the vehicle level because it must store overhead RAs and perform the required operations to be able to use them when visiting an area for which the vehicle has a matching overhead RA stored. However, it is important to emphasize that the solution does not require any kind of support from the network or other vehicles, and therefore, it is fully compatible with legacy GeoSAC systems. Because this optimization is local to a vehicle, vehicles implementing this overhearing-assisted optimization will benefit from the reduced IP address configuration time without impacting in any way the performance or operation of other vehicles.

\section{B. Overhearing Probability}

We first focus on analytically assessing how probable it is for a node to overhear an RA generated in a neighboring area. Let the overhearing probability $P_{\mathrm{oh}}$ be this probability. In this section, we derive an analytical expression for $P_{\mathrm{oh}}$.

We assume that deploying vehicular networks without dead zones uncovered by an RSU is economically inefficient (at least for nonurban and not densely populated scenarios). As we have discussed in Section II-B, vehicles form a self-organized multihop network in the ETSI TC ITS architecture. This multihop network is used to forward (at the ETSI GN layer) the RAs sent by an RSU, which flood its associated geographical area and thereby extend the effective coverage area of the RSU.

We use the following terminology throughout this paper (see Fig. 4). A vehicle that is located in the area $n-1$ and must be configured for the area $n$ (i.e., an adjacent area) is called a target. A forwarder (fwd) is a vehicle placed inside the area $n$ that is also within the radio coverage of the target. Let $D_{\mathrm{RSU}}$ be the distance between two adjacent RSUs, $R$ be the wireless communication range, $\beta$ be the vehicular density, and $v$ be the speed of the vehicles. ${ }^{4}$ A target node successfully overhears an RA when the forwarder receives an RA and forwards it to the target before it enters the area $n$. To be able to receive and forward an RA, the forwarder node should have connectivity

\footnotetext{
${ }^{4}$ We consider the speed of all vehicles to be fixed and constant for the sake of model simplicity. The simulation results we will present later prove that this simplification does not affect the validity of the conclusion of our analysis.
} 
with the RSU. Because this connectivity may involve multiple intermediate nodes (i.e., wireless hops), we call it multihop connectivity (mhc). Connectivity in ad hoc networks has been thoroughly studied, but vehicular networks have special characteristics (in particular, mobility patterns and range of speeds) that require specific analysis. Some studies have contributed to the characterization of connectivity in vehicular networks (for example, [22] and [23], which focus on intervehicle connectivity), but in this paper, we analyze connectivity from the particular viewpoint of address autoconfiguration, which requires characterizing the time needed until connectivity with an RSU (a fixed point in the road) is achieved.

Given a forwarder node and the RSU, $P_{\mathrm{mhc}}^{\mathrm{fwd}}$ is the probability of having mhe (that is, of having a chain of interconnected vehicles between fwd and the RSU so that messages can be exchanged between them).

We next model the probability $P_{\mathrm{oh}}$ of the target overhearing an RA originating at the area $n$. $P_{\text {oh }}$ can be modeled by splitting the original problem into two complementary subproblems. Having mhe between the RSU and the forwarder node is a necessary condition for successfully overhearing an RA. Without mhc, no RA overhearing (oh) is possible; therefore, oh $\subset$ mhc. From this condition, it is straightforward to derive that $O H \equiv O H \cap M H C$, i.e.,

$$
P_{\mathrm{oh}}=P(O H)=P(O H \cap M H C) .
$$

By applying the conditional probability theorem in (1), we have

$$
\begin{aligned}
P_{\mathrm{oh}} & =P(O H \cap M H C) \\
& =P(O H \mid M H C) P(M H C) \\
& =P_{\mathrm{oh} \mid \mathrm{mhc}} P_{\mathrm{mhc}}^{\mathrm{fwd}}
\end{aligned}
$$

where $P_{\mathrm{mhc}}^{\mathrm{fwd}}$ represents the probability of mhc for the forwarder. We first focus on $P_{\mathrm{oh} \mid \mathrm{mhc}}$.

Let $T_{n \mathrm{RA}}^{\mathrm{recv}}$ denote the time elapsed from the target vehicle being at distance $R$ from an adjacent area border to the time it receives an RA sent by an RSU of that adjacent area. This time can be split into two parts. The first part $T_{\text {fwd }}$ represents the time elapsed until the forwarder vehicle leaves the area $n-1$, enters the next area, and becomes ready to forward RAs to the target. The second part $T_{\mathrm{RA}}^{\mathrm{unsol}}$ is the time elapsed until an RA from the RSU is received under this mhc assumption. We assume that the time between two consecutive RAs sent by an RSU (or an access router in the case when the RSU is working in bridge mode) follows a uniform distribution between a minimum value MinRtrAdvInterval and a maximum value MaxRtrAdvInterval, which we refer to as $R_{m}$ and $R_{M}$, respectively [19]. By joining $T_{\mathrm{fwd}}$ and $T_{\mathrm{RA}}^{\mathrm{unsol}}$, we can express $T_{n \mathrm{RA}}^{\mathrm{recv}}$ as

$$
T_{n \mathrm{RA}}^{\mathrm{recv}}=T_{\mathrm{fwd}}+T_{\mathrm{RA}}^{\mathrm{unsol}} .
$$

Assuming exponentially distributed distances between cars [24], $T_{\mathrm{fwd}}$ follows an exponential distribution with parameter $\beta$; its probability density function (pdf) is given by

$$
f_{T_{\mathrm{fwd}}}(t)=\beta v e^{-\beta v t}, \quad t \geq 0 .
$$

Given that $T_{\mathrm{fwd}}$ and $T_{\mathrm{RA}}^{\mathrm{unsol}}$ are independent, the pdf of $T_{n \mathrm{RA}}^{\mathrm{recv}}$ is given by (5), shown at the bottom of the page.

Because an RA is overheard only if received by the vehicle before crossing the area border, and it takes $R / v$ seconds for the vehicle to reach the border, the probability $P_{\mathrm{oh} \mid \mathrm{mhc}}$ of overhearing an RA is given by

$$
P_{\mathrm{oh} \mid \mathrm{mhc}}=\int_{0}^{\frac{R}{v}} f_{T_{n \mathrm{RA}}^{\mathrm{recv}}}(t) d t .
$$

$P_{\mathrm{oh} \mid \mathrm{mhc}}$ represents the probability of overhearing an RA given that there exists mhe between the RSU and the forwarder vehicle.

We next model $P_{\text {mhc }}^{\mathrm{fwd}}$, which depends on the distance between the RSU and the forwarder node, the radio coverage of the wireless communication technology, and the vehicular density.

Given two nodes separated by a distance $D, P_{\mathrm{mhc}}(D)$ is the probability of having mhe between the two nodes. This probability depends on the distance between the two nodes $D$, the radio coverage of the wireless communication technology used $R$, and the vehicular density $\beta$. To have mhe between a forwarder node and the RSU, there should be a chain of connected vehicles (i.e., the distance between two consecutive vehicles must be less than or equal to $R$ ) between fwd and a vehicle within direct (single hop) radio coverage of the RSU. If $D_{\mathrm{mhc}}$ is the distance between these two nodes (see Fig. 4), then the probability of having mhe between the forwarder node and the RSU is given by $P_{\mathrm{mhc}}\left(D_{\mathrm{mhc}}\right)$.

The distance between two consecutive vehicles that are part of a connected multihop chain of vehicles (i.e., one in which the intervehicle gap is smaller than $R$ ) follows a truncated exponential distribution [25], i.e.,

$$
f_{t e}(d)= \begin{cases}\frac{\beta e^{-\beta d}}{1-e^{-\beta R}}, & 0<d<R \\ 0, & \text { otherwise. }\end{cases}
$$

The length of a multihop connected chain of $n+1$ vehicles $Y$ can be represented as the sum of $n$ independent truncated

$$
f_{T_{n \mathrm{RA}}^{\mathrm{recv}}}(t)=\left(f_{T^{\mathrm{fwd}}} * f_{T_{\mathrm{RA}}^{\text {unsol }}}\right)(t)= \begin{cases}\frac{2\left(1-\beta v e^{(-\beta v t)}\right)}{R_{M}+R_{m}}, & 0 \leq t \leq R_{m} \\ \frac{2\left(\beta v R_{M}-\beta v t+1-e^{-\beta v\left(t-R_{m}\right)}-\beta v\left(R_{M}-R_{m}\right) e^{-\beta v t}\right)}{\beta v\left(R_{M}^{2}-R_{m}^{2}\right)}, & R_{m}<t \leq R_{M} \\ \frac{2 e^{-\beta v\left(t-R_{M}\right)}-e^{\beta v R_{m}}-\beta v\left(R_{M}-R_{m}\right)}{\beta v\left(R_{M}^{2}-R_{m}^{2}\right)}, & t>R_{M}\end{cases}
$$


exponential variables. The pdf of $Y$ can be obtained by the method of characteristic functions [25]

$$
\begin{array}{r}
g_{Y}(y ; n)=\frac{(\beta b)^{n}}{(n-1) !} e^{-\beta y} \sum_{k=0}^{k_{0}}(-1)^{k}\left(\begin{array}{l}
n \\
k
\end{array}\right)(y-k R)^{n-1} \\
k_{0} R<y<\left(k_{0}+1\right) R
\end{array}
$$

where $k_{0}=0,1, \ldots, n-1$, and $b=\left(1-e^{-\beta R}\right)$.

Let $a=\left(k_{0}^{\prime}+c\right) R$, where $k_{0}^{\prime}$ is an integer, and $0 \leq c<1$. The cumulative distribution function (cdf) of $Y$ evaluated at $a$ is $G_{Y}(a ; n)=\int_{0}^{a} g_{Y}(y ; n) d y$, i.e.,

$$
\begin{aligned}
G_{Y}(a ; n)=\frac{1}{\left(1-e^{-\beta R}\right)^{-n}} & \sum_{k=0}^{k_{0}}(-1)^{k}\left(\begin{array}{l}
n \\
k
\end{array}\right) e^{-\beta k R} \\
& \times Q\left[2\left(k_{0}^{\prime}-k+c\right) R \beta, 2 n\right]
\end{aligned}
$$

where $Q[u, w]=P\left(\chi^{2}(w)<u\right)$, and $\chi^{2}(w)$ is a chi-square variable with $w$ degrees of freedom. Because the probability $P(i$ hops $)$ of having a connected chain of $i$ hops is given by $\left(1-e^{-\beta R}\right)^{i} e^{-\beta R}$, the pdf and cdf of the length $L$ of a connected multihop chain of vehicles can be derived using the total probability theorem, i.e.,

$$
\begin{aligned}
f_{L}(l) & =\sum_{i=0}^{\infty} P(i \text { hops }) g_{Y}(l ; i) \\
& =\sum_{i=0}^{\infty}\left(1-e^{-\beta R}\right)^{i} e^{-\beta R} g_{Y}(l ; i) \\
F_{L}(l) & =P_{L}(L \leq l) \\
& =\int_{0}^{l} f_{L}(u) d u \\
& =\sum_{i=0}^{\infty}\left(1-e^{-\beta R}\right)^{i} e^{-\beta R} G_{Y}(l ; i) .
\end{aligned}
$$

If we consider the maximum possible value of $D_{\mathrm{mhc}}$, which is given by $\left(D_{\mathrm{RSU}} / 2\right)-R$, then a pessimistic approximation of $P_{\mathrm{mhc}}^{\mathrm{fwd}}$ is given by

$$
P_{\mathrm{mhc}}^{\mathrm{fwd}}=P_{\mathrm{mhc}}\left(D_{\mathrm{mhc}}\right) \geq 1-F_{L}\left(\frac{D_{\mathrm{RSU}}}{2}-R\right) \text {. }
$$

The overhearing probability $P_{\text {oh }}$ can then be derived ${ }^{5}$ from (2) using (6) and (12), i.e.,

$$
\begin{aligned}
P_{\mathrm{oh}} & =P_{\mathrm{oh} \mid \mathrm{mhc}} P_{\mathrm{mhc}}^{\mathrm{fwd}} \\
\geq & \int_{0}^{\frac{R}{v}} f_{T_{n \mathrm{RA}}^{\mathrm{recv}}}(t) d t\left[1-F_{L}\left(\frac{D_{\mathrm{RSU}}}{2}-R\right)\right] .
\end{aligned}
$$

\footnotetext{
${ }^{5}$ This approximation is also pessimistic because we only consider the first opportunity to receive an unsolicited RA from the RSU, although there may be more than one before crossing the border.
}

We next describe the experiments that we performed to validate our mathematical model. Using a Matlab-based simulator, ${ }^{6}$ we conducted a large amount of experiments under different traffic conditions. The simulator implements the model described in this section, namely, vehicles distributed on a 1-D road, traveling at a fixed and constant speed, with an exponential intervehicular distance and a maximum wireless radio coverage, assuming an ideal wireless technology (no loses nor collisions and infinite bandwidth). We argue that although the simulator used for these tests does not consider a real wireless model, it is sufficient to assess if our mathematical analysis correctly matches the overhearing success probability in the scenario we have used in the analysis. In Section IV-A, when we present our analysis for the configuration time, we use a more advanced simulator $(\mathrm{OMNeT}++)$ that does include a complete wireless model.

Because traffic conditions play a critical role in the efficacy of wireless multihop communications in a vehicular environment, we studied several configurations to validate our model under different conditions. To limit the number of results presented in this paper, we have selected the following four scenarios, which mostly cover a wide spectrum of the potential traffic scenarios:

1) urban road: high vehicular density $(\beta=80 \mathrm{veh} / \mathrm{km})$ and low speed $(v=50 \mathrm{~km} / \mathrm{h})$;

2) city beltway: moderate vehicular density $(\beta=$ $50 \mathrm{veh} / \mathrm{km})$ and moderate speed $(v=80 \mathrm{~km} / \mathrm{h})$;

3) highway: low vehicular density $(\beta=35 \mathrm{veh} / \mathrm{km})$ and high speed $(v=120 \mathrm{~km} / \mathrm{h})$;

4) sparse: very low vehicular density $(\beta=10 \mathrm{veh} / \mathrm{km})$ and moderate speed $(v=100 \mathrm{~km} / \mathrm{h})$. Examples of this scenario are city beltways and highways at night, or secondary roads.

For each of these scenarios, we conducted experiments using two different values of the wireless coverage radio $R$ (150 and $300 \mathrm{~m}$ ) that cover possible IEEE 802.11-based technologies. Figs. 5 and 6 show the analysis and simulation results for $P_{\mathrm{oh}}$ versus different average intervals between RAs for different deployment scenarios (which are defined by the distance between RSUs $\left.D_{\mathrm{RSU}}\right)$. Note that different values of $D_{\mathrm{RSU}}$ are used depending on the value of $R$ because the coverage radius of the wireless technology has an impact on the deployment. For small values of $R$, it does not make any sense to deploy RSUs at long distances because the probability of having connectivity is low, and the number of hops is high (which has a negative impact on performance).

It can be noted from these results that our analytical model perfectly matches the results obtained via simulation. ${ }^{7}$ From these results, we can also observe that if a short range wireless technology is used $(R=150 \mathrm{~m})$, high overhearing success probabilities can be achieved only if the RSUs are configured with short inter-RA values $\left(T_{\mathrm{RA}}\right)$ and only for moderate density scenarios (urban, city beltway, and highway). For the case

\footnotetext{
${ }^{6}$ The code of the simulator is available at http://fourier.networks.imdea.org/ people/ marco_gramaglia/sims/GeoSAC-sim/.

${ }^{7}$ Note that we have also performed simulations under several other traffic conditions, and they supported the accuracy of our analytical model.
} 


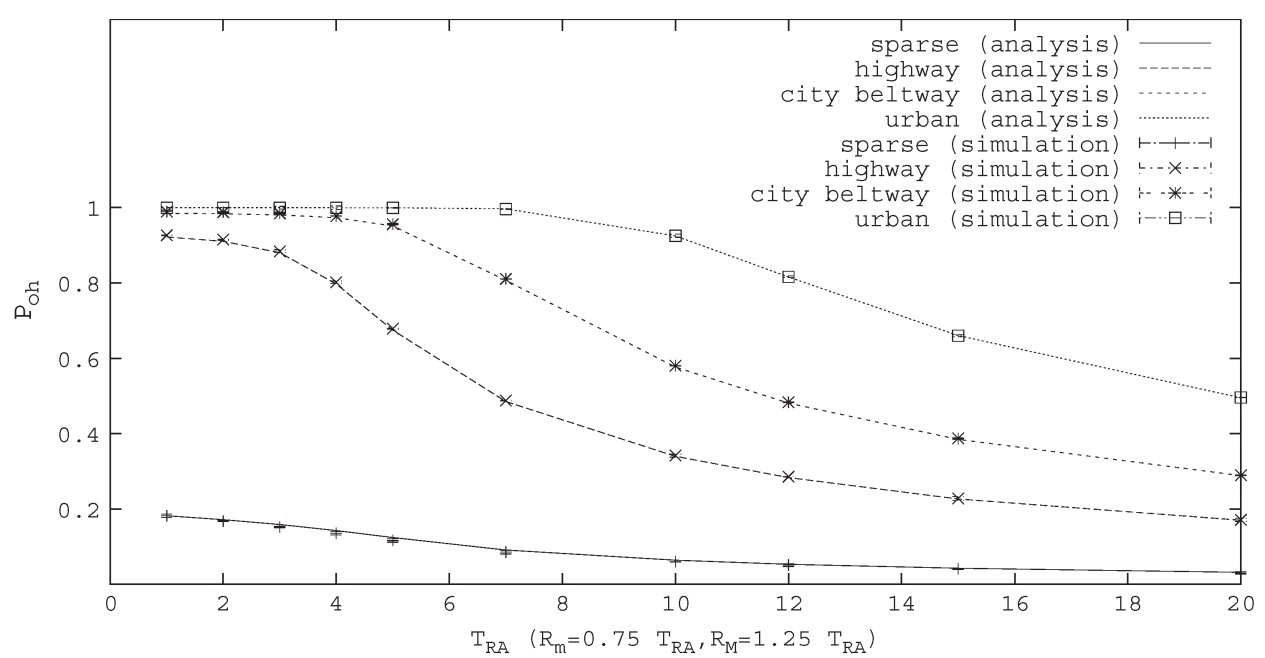

(a)

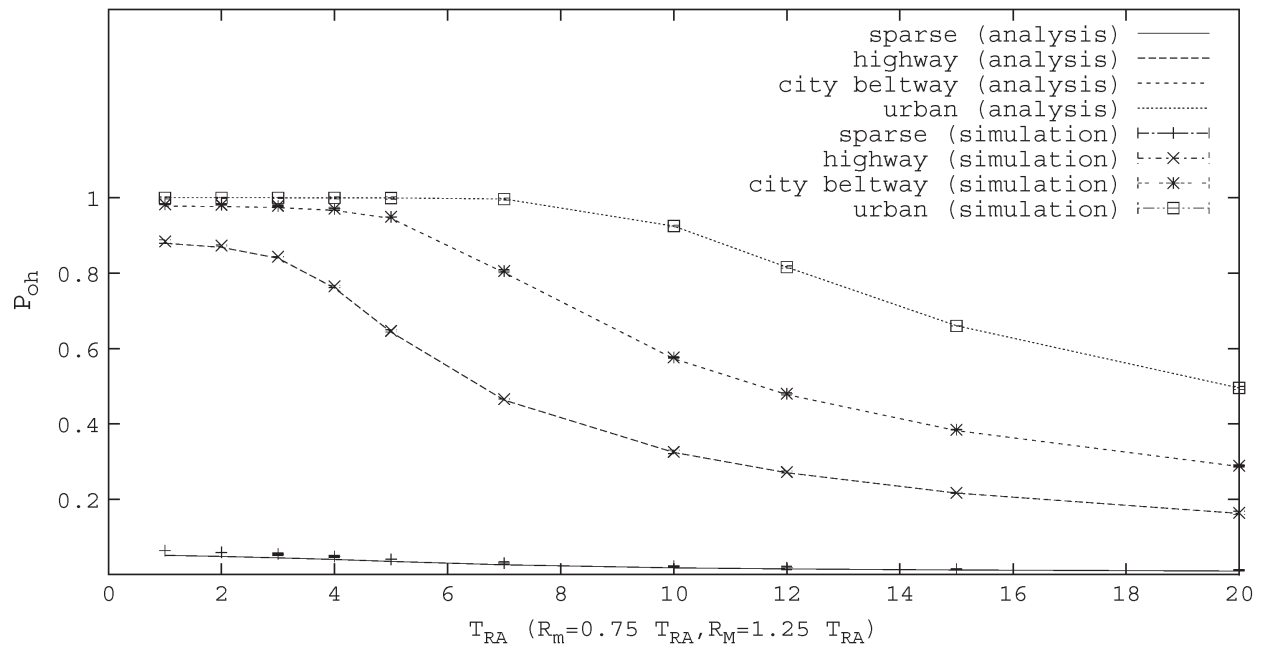

(b)

Fig. 5. Overhearing probability $R=150 \mathrm{~m}$. (a) $D_{\mathrm{RSU}}=1000 \mathrm{~m}$. (b) $D_{\mathrm{RSU}}=1500 \mathrm{~m}$.

of $R=300 \mathrm{~m}$ (i.e., for longer range wireless technologies), the probability of overhearing an RA increases (because it is very close to $100 \%$ ) without requiring too much resourceconsuming $T_{\mathrm{RA}}$ configuration settings. These results show that our overhearing-assisted optimization is feasible and can effectively reduce the IP address configuration time in most of the practical scenarios. The results also show that for $R=300 \mathrm{~m}$, there is a high probability of overhearing an RA, even in the sparse scenario. From this result, we can also observe the expected impact that $D_{\mathrm{RSU}}$ has on $P_{\mathrm{oh}}$, particularly with low vehicular densities.

\section{PERFormance AnAlysis And Evaluation}

In this section, we analyze the performance of our overhearing-assisted optimization by extending the analysis we have developed in the previous section to obtain an expression for the IP address configuration time when our optimization is enabled. This extension allows us to characterize the gains of our mechanism and compare them with the performance when overhearing is not enabled. Finally, because it is likely that a vehicular communications system will make use of an
IP mobility solution to transparently keep ongoing IP sessions alive, regardless of the movement of the vehicle (and the subsequent change of IP point of attachment and IP address), we also analyze the impact of the proposed optimization on the overall performance when IP mobility is enabled.

\section{A. IP Address Configuration Time}

The most obvious advantage of our overhearing optimization is the reduction of the average IP address configuration time because nodes that successfully overhear an RA from a neighboring area are able to immediately configure an address if entering into that area afterward. We define the GeoSAC IP address configuration time $T_{\text {conf }}$ as the time elapsed from when a vehicle crosses an area border until when it gets a valid IP address that can be used to send and receive packets while located in the new area. If our overhearing optimization is enabled, then we call the configuration time $T_{\text {conf }}^{\text {oh }}$. A node that overhears an RA from area $n$ while being at area $n-1$ and then enters into area $n$ does not need to wait for any signaling before configuring and starting to use an IP address; therefore, $T_{\text {conf }}^{\mathrm{oh}}=0 \mathrm{~s}$ in this case. Depending on the deployment scenario and traffic conditions, 


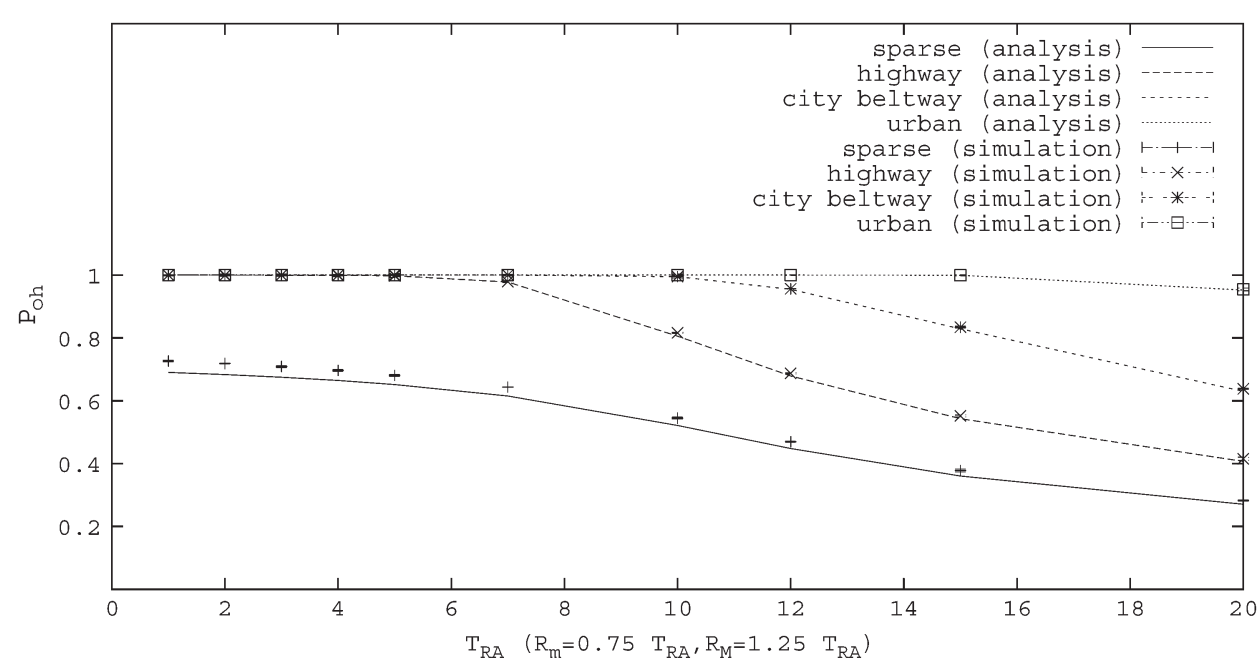

(a)

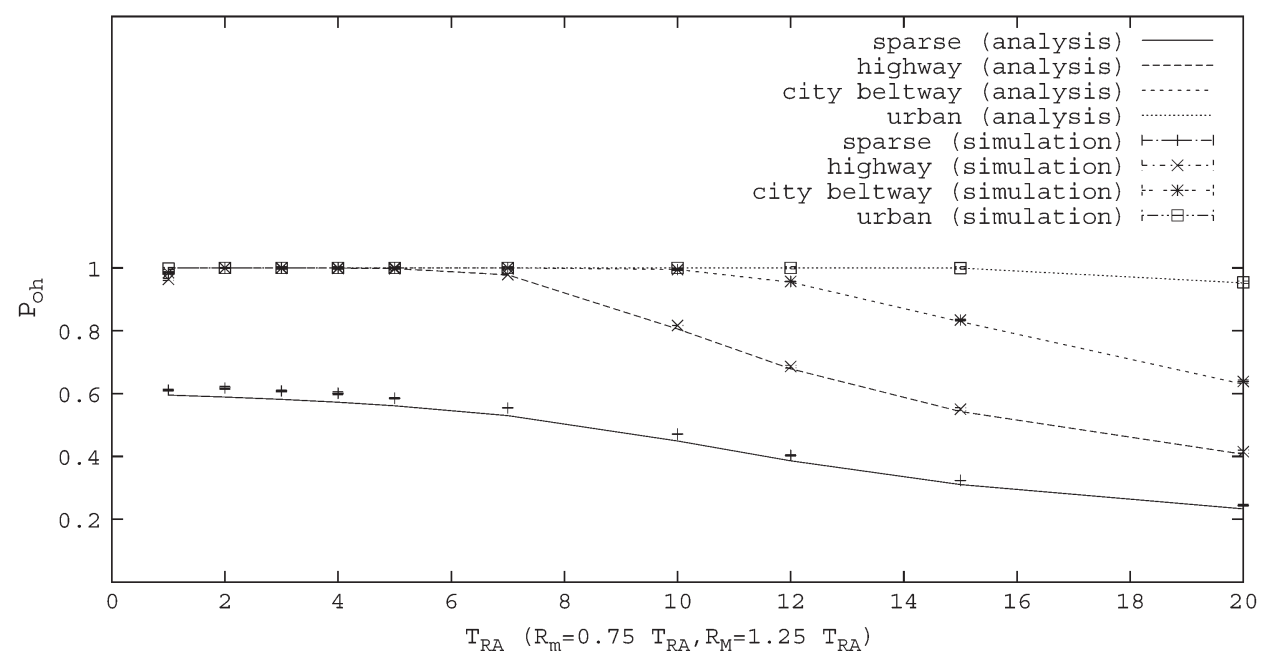

(b)

Fig. 6. Overhearing probability $R=300 \mathrm{~m}$. (a) $D_{\mathrm{RSU}}=1500 \mathrm{~m}$. (b) $D_{\mathrm{RSU}}=2000 \mathrm{~m}$.

it is not always possible for a node to successfully overhear an RA from an area that the node is about to enter. We performed a mathematical analysis that models $T_{\text {conf }}^{\mathrm{oh}}$. This model allowed us to evaluate the gains obtained by using our optimization, focusing first on the IP address configuration time reduction compared with the case where plain (i.e., no optimization enabled) GeoSAC is used.

To make the analysis easier to follow, we have divided it into different parts, each of them corresponding to a different configuration scenario in which a node might be involved. This approach allows us to derive the average configuration time of GeoSAC when our overhearing optimization is enabled. There are basically four possible situations that have to be considered:

1) There exists a forwarder node (located in area $n$ ) in the wireless coverage of the target node (which is located in area $n-1$ ), there is mhc between the forwarder node and the RSU of area $n$, and the RSU sends an unsolicited RA while the target node has not yet crossed the area border (see Fig. 4). In this case (corresponding to overhearing success), the configuration time is $0 \mathrm{~s}$.
2) This case is identical to 1), but an RA from area $n$ is not received by the target node while still in area $n-1$. In this case, the configuration time is equal to the time elapsed from the target node crossing the area border until it gets an RA from the RSU. The average of this time $\bar{T}_{\text {wait RA }}$ is given by

$\bar{T}_{\text {wait RA }}=\frac{\int_{R / v}^{\infty} t\left(f_{T^{\mathrm{fwd}}}^{\mathrm{FWD}} * f_{T_{\mathrm{RA}}^{\mathrm{unsol}}}\right)(t) d t}{\int_{R / v}^{\infty}\left(f_{T^{\mathrm{fwd}}}^{\mathrm{FWD}} * f_{T_{\mathrm{RA}}^{\mathrm{unsol}}}\right)(t) d t}-\frac{R}{v}$

where $f_{T^{\text {fwd }}}^{\mathrm{FWD}}$ is given by

$$
f_{T^{\mathrm{fwd}}}^{\mathrm{FWD}}(t)= \begin{cases}f_{T^{\mathrm{fwd}}}(t)=\beta v e^{-\beta v t}, & 0 \leq t \leq R / v \\ 0, & \text { otherwise }\end{cases}
$$

3) In this case, there is no forwarder node within the radio range of the target node (see Fig. 7). The target node has to get direct (i.e., one hop) connectivity from the RSU first and then wait for the next unsolicited RA. 


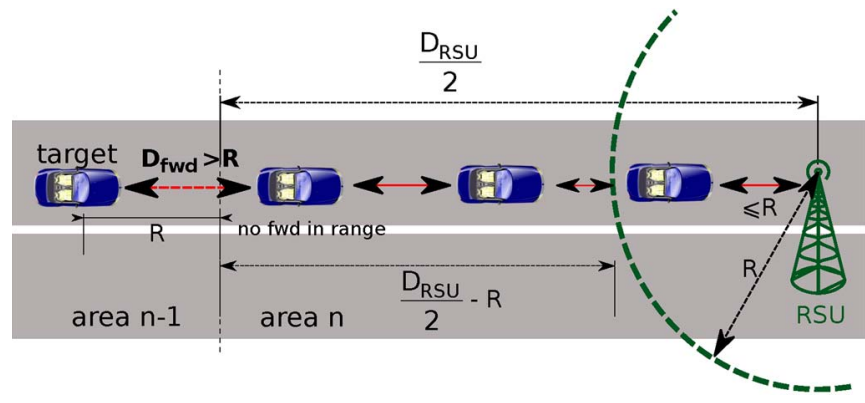

Fig. 7. No forwarder node within range of the target vehicle.

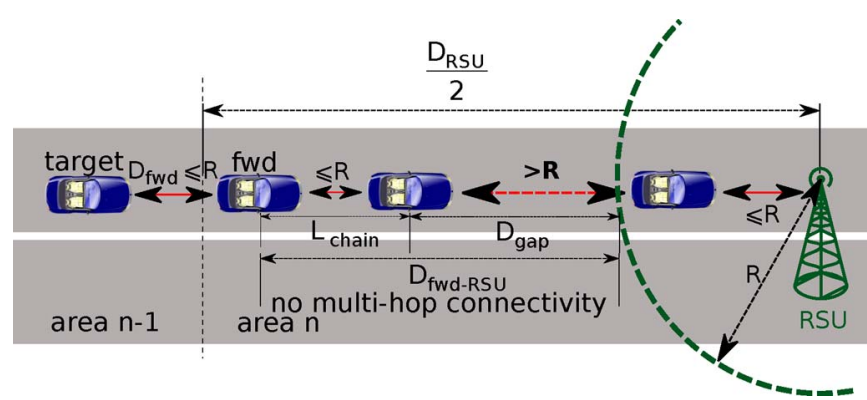

Fig. 8. No mhc available between the target vehicle and the RSU.

The average configuration time for this case $\bar{T}_{\text {no FWD }}$ is, therefore, given by

$$
\bar{T}_{\text {noFWD }}=\frac{D_{\mathrm{RSU}} / 2-R}{v}+\bar{T}_{\mathrm{RA}}^{\text {unsol }} .
$$

4) In this case, there exists a forwarder node in wireless coverage of the target node at area $n-1$, but there is no mhc between the forwarder and the RSU of area $n$ (see Fig. 8). The configuration time is the time required for the forwarder node to get connectivity to the RSU (as the forwarder node moves toward the RSU, the probability of having connectivity with the RSU increases) plus the time until an RA is sent. Here, we know that the length of the chain is shorter than $D_{\mathrm{RSU}} / 2-R$. By finding the average length of a multihop chain, we can obtain the average size of the gap between the last vehicle of the chain and the RSU coverage area border ( $\bar{D}_{\text {gap }}$ in Fig. 8). The final $\bar{T}_{\text {conf }}$ for these cases is computed by adding the average delay for getting an unsolicited RA $\left(\bar{T}_{\mathrm{RA}}^{\text {unsol }}\right)$.

We must first calculate the average distance between the target and the forwarder. By definition, the forwarder is the farthest vehicle that can relay an RA to the target because it is placed at most $R$ meters away from it. Thus, for a given density and coverage radius, the forwarder is placed at $\bar{D}_{\mathrm{fwd}}$. To calculate this value, we introduce $\bar{L}_{g_{Y}(y ; k)}$, the average length of a chain composed of a generic set of vehicles that are exponentially distributed with parameter $\beta$ and no longer than a maximum value $R$, i.e.,

$$
\bar{L}_{g_{Y}(y ; k)}=\int_{0}^{R} y g_{Y}(y ; k) d y .
$$

The probability of a chain composed by $k+1$ vehicles being shorter than $R$ is $\left(1-e^{-\beta R}\right)^{k} e^{-\beta R} G_{Y}(R, k)$. From this probability, we can calculate the average distance of the farthest vehicle within $R$ meters from the target (i.e., the forwarder) as follows:

$$
\bar{D}_{\mathrm{fwd}}=\frac{\sum_{0}^{\infty}\left(1-e^{-\beta R}\right)^{k} e^{-\beta R} G_{Y}(R, k) \bar{L}_{g_{Y}(y ; k)}}{\sum_{0}^{\infty}\left(1-e^{-\beta R}\right)^{k} e^{-\beta R} G_{Y}(R, k)} .
$$

The average distance between the forwarder and the coverage area of the RSU is given by

$$
\bar{D}_{\mathrm{fwd}-\mathrm{RSU}}=\frac{D_{\mathrm{RSU}}}{2}-R-\bar{D}_{\mathrm{fwd}} .
$$

The average length of a chain of vehicles that is shorter than $\bar{D}_{\text {fwd-RSU }}$ is

$$
\bar{L}_{\text {chain }}=\frac{\sum_{0}^{\infty}\left(1-e^{-\beta R}\right)^{k} e^{-\beta R} G_{Y}\left(\bar{D}_{\mathrm{fwd}-\mathrm{RSU}}, k\right) \bar{L}_{g_{Y}(y ; k)}}{\sum_{0}^{\infty}\left(1-e^{-\beta R}\right)^{k} e^{-\beta R} G_{Y}\left(\bar{D}_{\mathrm{fwd}-\mathrm{RSU}}, k\right)} .
$$

Therefore, the average gap length is given by

$$
\bar{D}_{\text {gap }}=\bar{D}_{\text {fwd-RSU }}-\bar{L}_{\text {chain }}
$$

and from this result, we can calculate the time required to configure an IP address. In this case

$$
\bar{T}_{\text {no MHC }}=\frac{\bar{D}_{\text {gap }}}{v}+\bar{T}_{\mathrm{RA}}^{\text {unsol }} .
$$

To calculate the probability of each of the four identified situations occurring, we performed some probability calculations, i.e.,

$$
\begin{gathered}
P(F W D)[P((O H \mid M H C) \mid F W D) \\
+P((\overline{O H} \mid M H C) \mid F W D)] \\
+P(\overline{\mathrm{FWD}})[P((O H \mid M H C) \mid \overline{\mathrm{FWD}}) \\
+P((\overline{\mathrm{OH}} \mid M H C) \mid \overline{\mathrm{FWD}})]=1
\end{gathered}
$$

where $P(F W D)$ is the probability of a forwarder node existing (i.e., being within $R$ meters from the target node). Because $P((O H \mid M H C) \mid \overline{\mathrm{FWD}})=0$ (it is not possible to have overhearing success if there is no forwarder node $)$ and $P((\overline{\mathrm{OH}}$ $M H C) \mid \overline{\mathrm{FWD}}$ ) $=1$ (if there is no forwarder node, it is impossible to overhear an RA), we can further expand (22) as follows:

$$
\begin{aligned}
P(( & O H \mid M H C) \cap F W D) \\
& +P((\overline{O H} \mid M H C) \cap F W D)+P(\overline{\mathrm{FWD}}) \\
= & P(O H \mid M H C)+P((\overline{O H} \mid M H C) \cap F W D) \\
& +P(\overline{\mathrm{FWD}}) \\
= & P(O H \mid M H C) P(M H C) \\
& +P((\overline{O H} \mid M H C) \cap F W D) P(M H C) \\
& +P(\overline{\mathrm{FWD}}) P(M H C) \\
& +[1-P(\overline{\mathrm{FWD}})] P(\overline{M H C})
\end{aligned}
$$




$$
\begin{aligned}
& +P(\overline{\mathrm{FWD}}) P(\overline{M H C}) \\
= & P(O H)+P((\overline{O H} \mid M H C) \cap F W D) P(M H C) \\
& +P(\overline{\mathrm{FWD}})[P(M H C)+P(\overline{M H C})] \\
& +P(F W D) P(\overline{M H C}) \\
= & P(O H)+P((\overline{O H} \mid M H C) \cap F W D) P(M H C) \\
+ & P(\overline{\mathrm{FWD}})+P(F W D) P(\overline{M H C})=1
\end{aligned}
$$

in which we have also applied some properties of conditional probabilities. The goal of this analysis was to determine the probabilities of each of the four different situations that we previously identified. Using (13)-(21) and (23), we can obtain an expression for the average IP address configuration time when the overhearing optimization is enabled, i.e.,

$$
\begin{aligned}
\bar{T}_{\mathrm{conf}}^{\mathrm{oh}}= & P(O H) 0+P((\overline{O H} \mid M H C) \cap F W D) \\
& \times P(M H C) \bar{T}_{\text {wait RA }}+P(\overline{\mathrm{FWD}}) \bar{T}_{\mathrm{no} \mathrm{FWD}} \\
& +P(F W D) P(\overline{M H C}) \bar{T}_{\mathrm{no} \mathrm{MHC}}
\end{aligned}
$$

where

$$
P((\overline{O H} \mid M H C) \cap F W D)=\int_{R / v}^{\infty}\left(f_{T^{\mathrm{fwd}}}^{\mathrm{FWD}} * f_{T_{\mathrm{RA}}^{\mathrm{unsol}}}\right)(t) d t
$$

$$
P(\overline{\mathrm{FWD}})=1-P(F W D)=1-e^{-\beta R}
$$

$$
\begin{aligned}
P(M H C) & =P_{\mathrm{mhc}}^{\mathrm{fwd}}=P_{\mathrm{mhc}}\left(D_{\mathrm{mhc}}\right) \\
& \geq 1-F_{L}\left(\frac{D_{\mathrm{RSU}}}{2}-R\right) .
\end{aligned}
$$

We next validated our mathematical analysis by means of simulation. To consider more realistic wireless conditions, we implemented our overhearing optimization for $\mathrm{GeoSAC}^{6}$ using OMNeT++ and the Mixim framework. Mixim ${ }^{8}$ is a framework for a wireless ad hoc network for the OMNeT++ simulator. ${ }^{9}$ It provides the 802.11 MAC layer and many physical layer models (including the widely accepted path loss, shadowing, and large- and small-scale fading models [26]-[28]). The simulation scenario consists of a road segment where vehicles travel within a homogeneous flow. The vehicles' starting positions are generated using an exponential distribution. The speed and density are defined by the type of scenario: urban, city beltway, highway, and sparse; therefore, the number of nodes involved in the simulation changes depending on the vehicular density. At the end of the road segment, nodes enter a GeoSAC area $\left(D_{\mathrm{RSU}}\right.$ meters long), where an RSU is placed half-way $\left(D_{\mathrm{RSU}} / 2\right.$ from the area border). The vehicles are equipped with a standard 802.11g MAC layer with a bit rate of $6 \mathrm{Mb} / \mathrm{s}$. When the

\footnotetext{
${ }^{8}$ The code of this simulator is available at http://fourier.networks.imdea.org/ people/ marco_gramaglia/sims/GeoSAC-sim/

${ }^{9} \mathrm{http} / / /$ www.omnetpp.org/
}

TABLE I

Simulation PARAMETERS

\begin{tabular}{|l|c|c|}
\hline Scenario & Speed $[\mathrm{km} / \mathrm{h}]$ & Density $[\mathrm{veh} / \mathrm{km}]$ \\
\hline Urban & 50 & 80 \\
\hline Beltway & 80 & 50 \\
\hline Highway & 120 & 35 \\
\hline MAC Layer & \multicolumn{2}{|c|}{$802.11 \mathrm{~g}$} \\
\hline Bitrate & \multicolumn{2}{|c|}{$6 \mathrm{Mb} / \mathrm{s}$} \\
\hline
\end{tabular}

simulation starts, the vehicles are first excluded from the results' recollection because they were already located inside the GeoSAC area, but they are needed to build the multihop chain and to allow the subsequent vehicles to be configured. When a node receives the first RA after crossing the area border, its configuration time is recorded. Each simulation is run 20 times using the same topology with a different seed, and for each parameter set, 50 different topologies are generated. The results are averaged on a population of at least $1000 \times n$ Cars values, where $n$ Cars depends on the chosen vehicular density. Because the road segment length is $15 \mathrm{~km}$, in the worst case, this value is approximately 150 . The parameters used in the simulations are summarized in Table I.

Figs. 9 and 10 show the obtained results using the OMNeT++-based simulation. In this case, we used two different values of $R: 1$ ) $R=225 \mathrm{~m}$, which is the average coverage value between two wireless nodes in the OMNeT++ simulation, when configured as in our experiments (see Fig. 10), and 2) $R=150 \mathrm{~m}$, which is one of the values we used in the previous simulations that helped to better understand the performance of our optimization when the probability of having mhe is lower (see Fig. 9). In addition to the simulation and analytical results, we also depict the best possible value for the IP address configuration time that plain GeoSAC could achieve [7]. This value, which corresponds to the optimistic nonideal assumption that there is always mhc between an unconfigured node and the RSU, is equal to $\bar{T}_{\mathrm{RA}}^{\text {unsol }}$. The simulation results validate our mathematical analysis. ${ }^{10}$ They show that for nonsparse scenarios and values of $T_{\mathrm{RA}}$ between 1 and $20 \mathrm{~s}$, the average IP address configuration time is always shorter than the best possible value that could be obtained with plain GeoSAC (i.e., without our optimization enabled). In addition, the improvement provided by overhearing optimization is quite large (the average configuration time is close to zero for several values of $T_{\mathrm{RA}}$ ). With $R=150 \mathrm{~m}$, the optimization does not provide any improvement in the sparse scenario. Note that the IP address configuration time displayed in Figs. 9 and 10 is sometimes larger for the sparse scenario than for plain GeoSAC under the best possible conditions. This is because the value for GeoSAC without overhearing is the value that would be achieved if there is always mhc between the vehicle and the RSU, which is far from true in sparse scenarios. In those scenarios, the IP address configuration time with and without our optimization enabled would be very similar. We also want to mention that the use case scenarios in which it makes sense to deploy an IP multihop network to connect vehicles to the Internet ranges

\footnotetext{
${ }^{10}$ We also performed a large number of experiments using our Matlab-based simulator that also validated our analysis. We do not show them because of space constraints.
} 


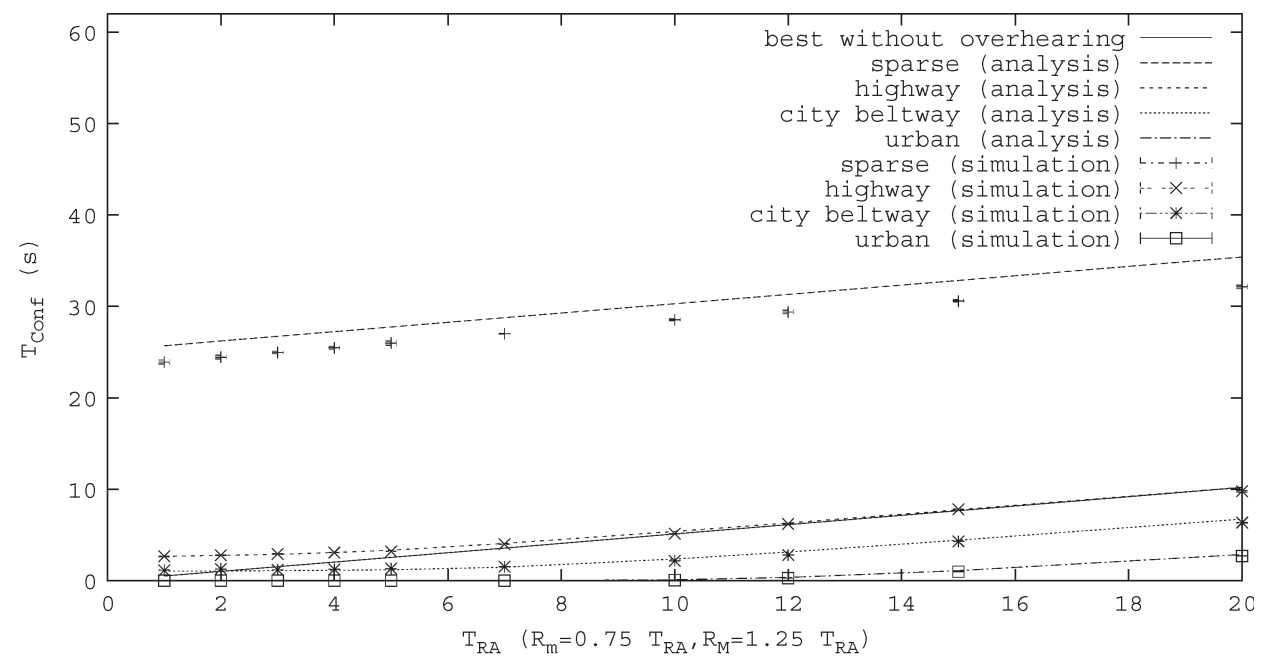

(a)

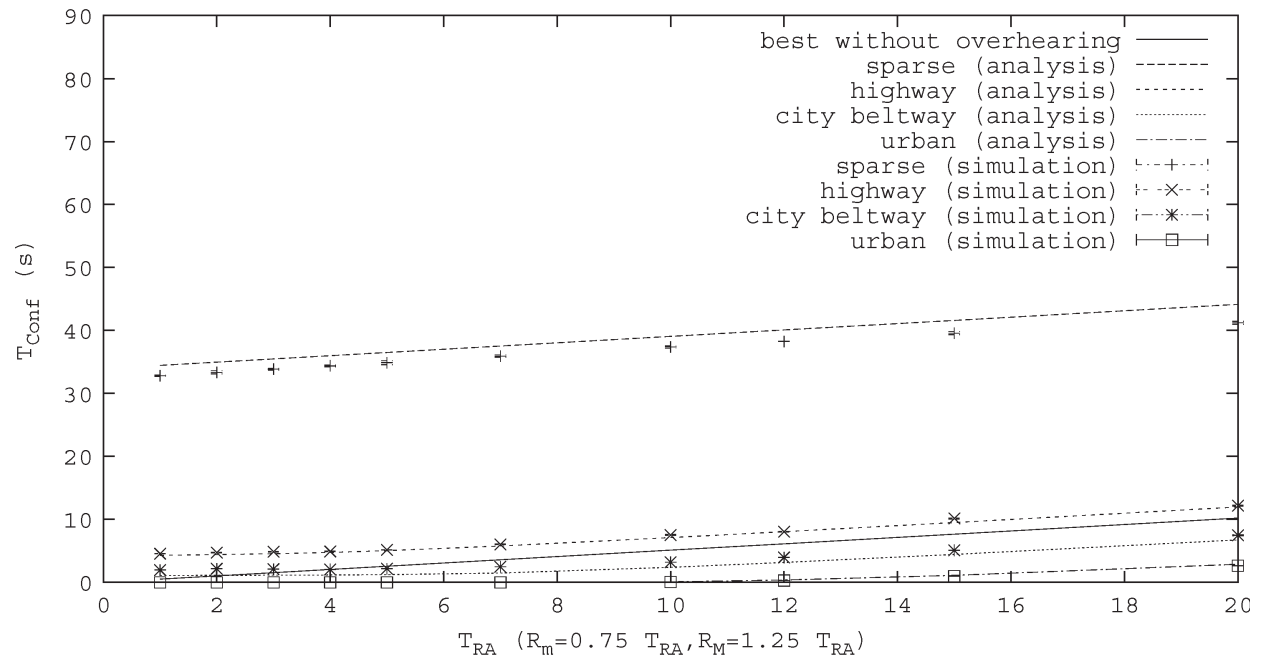

(b)

Fig. 9. IP address configuration time (analysis and OMNeT++ simulation results) $R=150 \mathrm{~m}$. (a) $D_{\mathrm{RSU}}=2000 \mathrm{~m}$. (b) $D_{\mathrm{RSU}}=2500 \mathrm{~m}$.

from moderate to high vehicular density networks (with proper RSU placement). While providing a more effective support for sparse networks could be possible, it would introduce a lot of complexity and computational costs, while bringing limited benefits, given the low connectivity level that vehicles experience in those scenarios.

Our last experiment to validate our analysis and the effectiveness of our overhearing optimization consisted of evaluating the configuration time using vehicular traces from a real road in Madrid, Spain. Using the OMNeT++ simulator, we assumed the position and speed of vehicles in a real road from traffic traces, evaluated the overhearing probability [see Fig. 11(a)], and measured the GeoSAC configuration time. The traces were taken at the three-lane city beltway M40 in Madrid and accounted for the traffic from 8:30 to 9:00 A.M. (which can be considered as near the rush hour). The total number of samples was 2560 . For each sample, we have a time stamp and a vehicle speed. We considered the measurement point to be the border between two geographical areas and assumed that each vehicle maintains the same speed while traversing the area. For our simulation environment, we fixed the distance between two
RSUs at $2000 \mathrm{~m}$. Fig. 11(b) shows the results obtained from the simulation and our mathematical analysis [see (24)]. In our mathematical model, we used the vehicular density calculated from the traces $(\beta=54 \mathrm{veh} / \mathrm{km})$ and the average speed $(v=$ $95 \mathrm{~km} / \mathrm{h}$ ). As can be observed from Fig. 11(b), the results using real vehicular traces confirm our previous findings, showing how overhearing optimization is able to significantly reduce the IP address configuration time.

It is worth highlighting the influence of $T_{\mathrm{RA}}$ on the performance gain provided by overhearing optimization. First, with relatively low values of $T_{\mathrm{RA}}$ (up to $8 \mathrm{~s}$ ), the average configuration time is generally quite low (zero or close to zero) for all the scenarios. Second, increasing $T_{\mathrm{RA}}$ gradually impacts the performance by increasing the average configuration time without abrupt changes. This result basically means that the system can be configured to meet certain performance and signaling overhead requirements by setting up the correct $T_{\mathrm{RA}}$ values on the RSUs. Although shorter $T_{\mathrm{RA}}$ values provide better performance, there is an additional overhead cost that must be considered. The next section explores in further detail the tradeoff between signaling overhead and configuration time. 


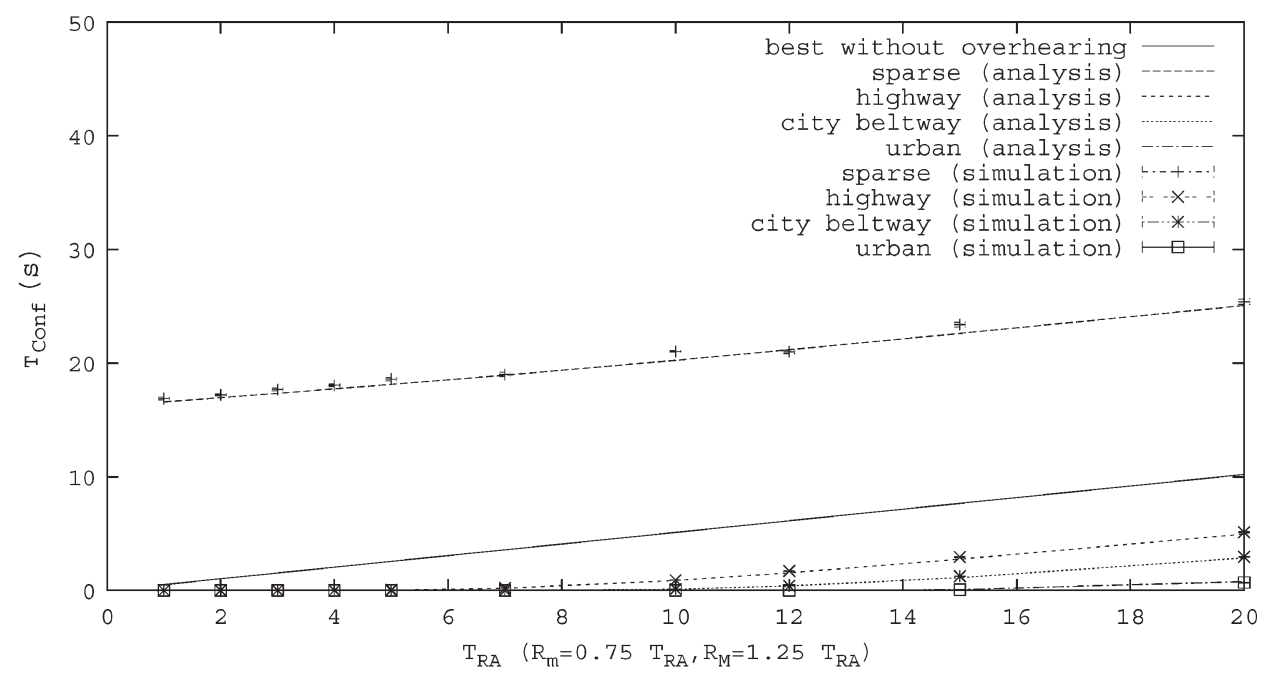

(a)

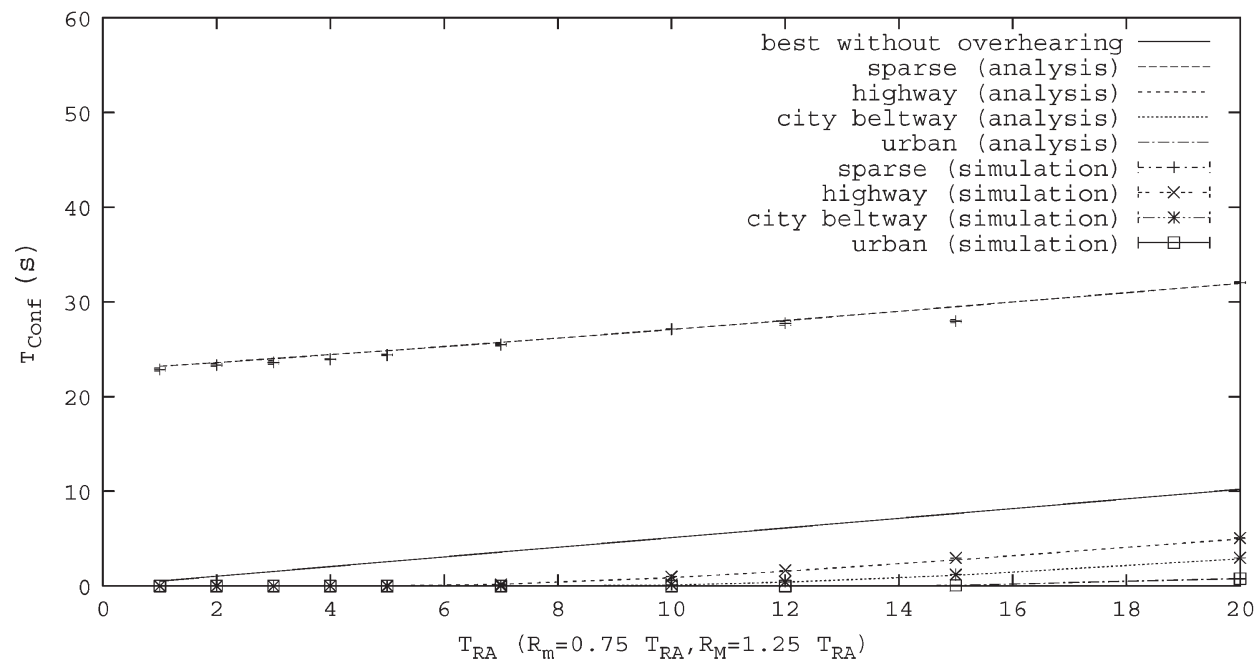

(b)

Fig. 10. IP address configuration time (analysis and OMNeT++ simulation results) $R=225 \mathrm{~m}$. (a) $D_{\mathrm{RSU}}=2500 \mathrm{~m}$. (b) $D_{\mathrm{RSU}}=3000 \mathrm{~m}$.

\section{B. Signaling Savings}

We have demonstrated in the previous subsection that enabling our overhearing-assisted optimization greatly improves the GeoSAC performance in terms of IP address configuration time. However, our optimization can also, by sending unsolicited RAs less frequently, be used to reduce the signaling required to achieve a certain target configuration time.

From the simulations we have performed, we can obtain what the minimum RA frequency required to achieve this target configuration time is with and without our overhearing optimization enabled. In Table II, we provide some results (for $R=225 \mathrm{~m}$ ) to help evaluate the signaling savings that can be obtained. In the urban scenario, for example, a value of $T_{\mathrm{RA}}=15 \mathrm{~s}$ is sufficient to obtain a shorter configuration time than the value obtained by plain GeoSAC with $T_{\mathrm{RA}}=2 \mathrm{~s}$. For the city beltway and highway scenarios, the results are similar. However, the difference in performance decreases as the vehicular density decreases, and the probability of overhearing success decreases. In the highway scenario, for example, $T_{\mathrm{RA}}$ can be increased by up to $10 \mathrm{~s}$ if the goal is to achieve a better performance than with plain GeoSAC and $T_{\mathrm{RA}}=2 \mathrm{~s}$.

\section{Impact on the Handover}

GeoSAC was designed as a mechanism to enable vehicles in a self-configured VANET to obtain a valid IP address. Getting an IP address is just one of the functionalities needed to connect vehicles to the Internet. As discussed in Section I, routing and mobility support are also important components. Routing within a VANET is independent of the IP addressing in the ETSI TC ITS system architecture; therefore, the performance of the IP address autoconfiguration protocol has no impact on the routing functionality. However, that lack of impact is not the case for IP mobility management protocols, in which the speed of IP address acquisition has a direct impact on the overall performance of the mobility solution and, thus, on the IP connectivity.

Mobile IPv6 [19] is the standardized solution for providing IP mobility support. The NEMO basic support protocol [15] is an extension to mobile IPv6 for enabling the movement of complete networks instead of just single hosts. Note that it is likely that vehicles will need a NEMO solution because cars are expected to be equipped with many devices with connectivity requirements. Every time a vehicle changes its IP point of attachment, the mobility protocol signals the movement to a 


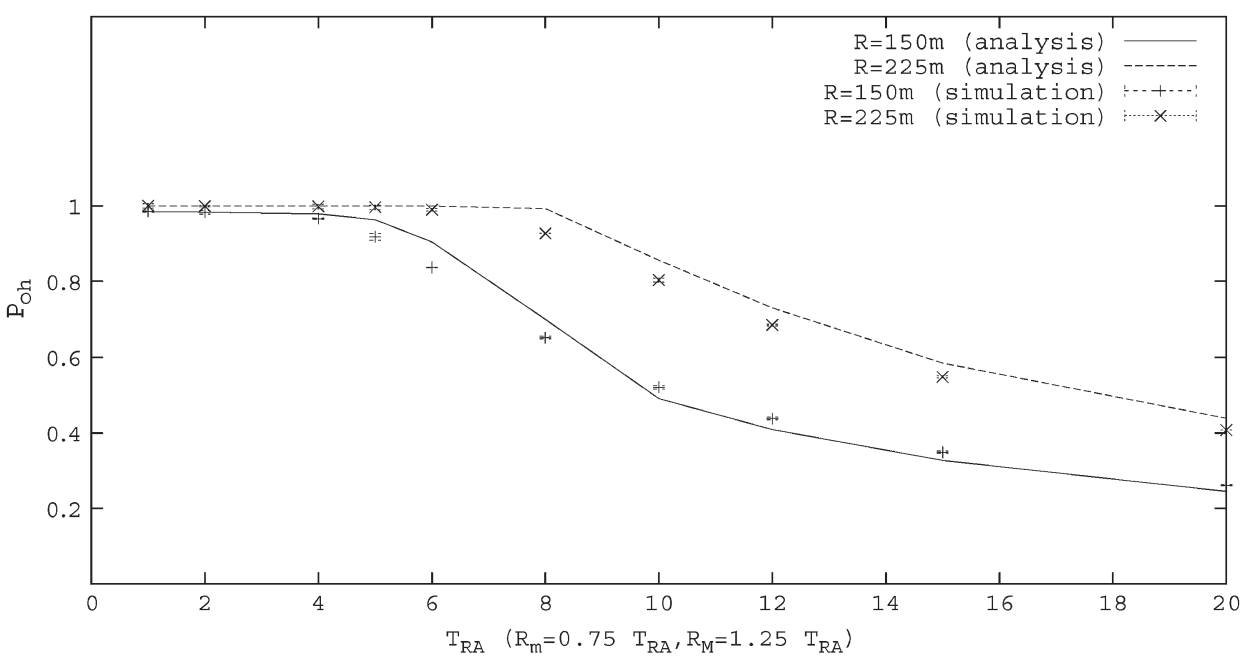

(a)

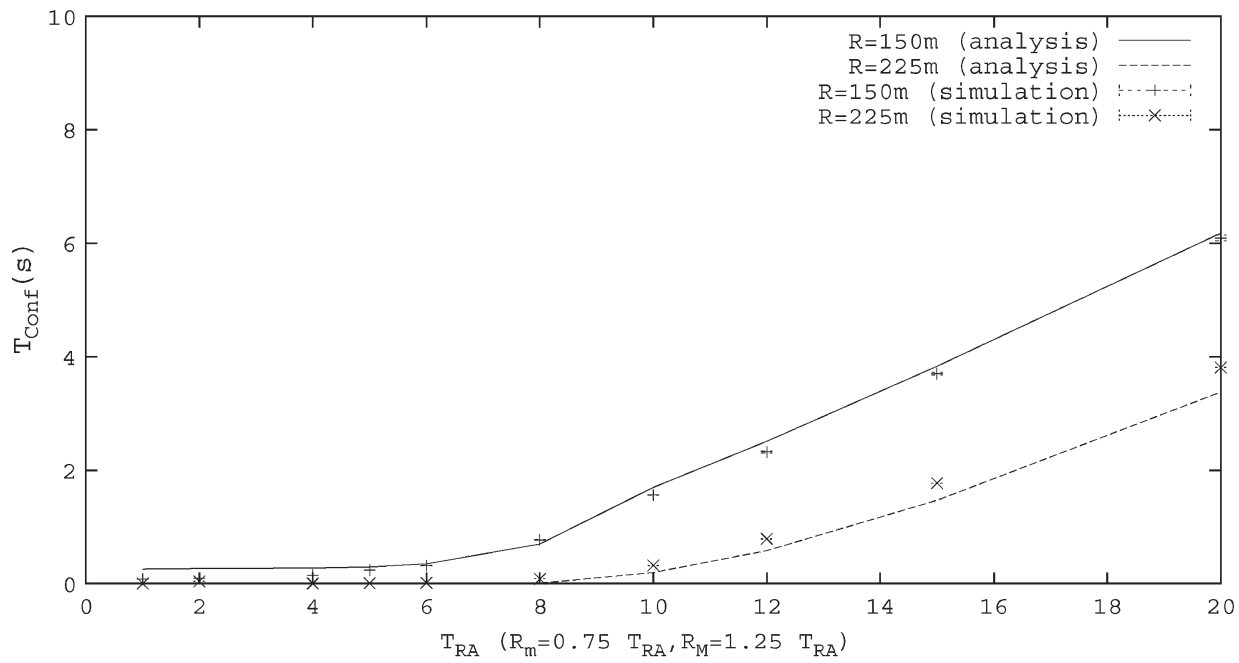

(b)

Fig. 11. Analysis and simulation with OMNeT++ and real vehicular traces. (a) Overhearing probability. (b) Overhearing-enabled GeoSAC configuration time.

central entity called the HA, which keeps track of the current location of the mobile node. Every time the vehicle moves, there is an interruption time (called handover latency) during which the vehicle cannot send or receive packets until all the mobility operations are completed. This handover time can be expressed as

$$
T_{h o}=T_{\mathrm{MD}}+T_{\mathrm{conf}}+R T T(\mathrm{veh}, \mathrm{HA})
$$

where $T_{\mathrm{MD}}$ represents the time required by the vehicle to detect that it has changed its point of attachment, $T_{\text {conf }}$ represents the time required to configure a valid IP address at its new location, and $R T T$ (veh, HA) represents the round trip time between the vehicle and the corresponding HA. $T_{\mathrm{MD}}=0 \mathrm{~s}$ because, as with GeoSAC, a change in its point of attachment corresponds to a change of geographical area, which the vehicle can easily detect by monitoring its GPS coordinates. RTT(veh, HA) depends on the distance between the vehicle and its HA, and it is typically on the order of milliseconds. $T_{\text {conf }}$ is the main component in (28). Therefore, reducing the address configuration time has a clear impact on the overall performance.
Table III shows the average GeoSAC handover delay $\left(\bar{T}_{\text {ho }}\right)$ with and without the overhearing optimization enabled for the city beltway scenario (with $R=225 \mathrm{~m}$ ) and the different components used in the calculation of this delay. Because the mobility signaling delay depends on the RTT between the vehicle and its mobility anchoring point (its HA), and because this delay depends on the location of these two entities, three different values of $R T T$ (veh, HA) were used in this analysis, representing "local," "regional," and "continental" delays [we used measurements taken from the ping end-to-end reporting (PingER) project ${ }^{11}$ ]. For each of these delay values, different RA intervals were used. The savings in the overall handover delay achieved by the use of the overhearing optimizations are more than $70 \%$ in the scenarios analyzed. In addition, the absolute handover latency values that are obtained when the overhearing optimization is enabled are small enough to even enable applications with certain latency constraints to be deployed in a vehicular network.

\footnotetext{
${ }^{11}$ http://www-iepm.slac.stanford.edu/pinger/
} 
TABLE II

AdDress Configuration Time $(R=225 \mathrm{~m})$

\begin{tabular}{|c|c|c|c|c|c|c|}
\hline$D_{R S U}(\mathrm{~m})$ & $v(\mathrm{~km} / \mathrm{h})$ & $\beta(\mathrm{veh} / \mathrm{km})$ & $\bar{T}_{R A}$ & $\bar{T}_{\text {conf }}$ & $\bar{T}_{\text {conf }}^{\text {oh }}$ & Saving \\
\hline \multirow{10}{*}{2000} & \multirow{10}{*}{50} & \multirow{10}{*}{80} & $1 \mathrm{~s}$ & $0.51 \mathrm{~s}$ & $0 \mathrm{~s}$ & $100 \%$ \\
\hline & & & $2 \mathrm{~s}$ & $1.02 \mathrm{~s}$ & $0 \mathrm{~s}$ & $100 \%$ \\
\hline & & & $3 \mathrm{~s}$ & $1.53 \mathrm{~s}$ & $0 \mathrm{~s}$ & $100 \%$ \\
\hline & & & $4 \mathrm{~s}$ & $2.04 \mathrm{~s}$ & $0 \mathrm{~s}$ & $100 \%$ \\
\hline & & & $5 s$ & $2.55 \mathrm{~s}$ & $0 \mathrm{~s}$ & $100 \%$ \\
\hline & & & $7 \mathrm{~s}$ & $3.57 \mathrm{~s}$ & $0 \mathrm{~s}$ & $100 \%$ \\
\hline & & & $10 \mathrm{~s}$ & $5.10 \mathrm{~s}$ & $0.06 \mathrm{~s}$ & $98.7 \%$ \\
\hline & & & $12 \mathrm{~s}$ & $6.13 \mathrm{~s}$ & $0.27 \mathrm{~s}$ & $95.5 \%$ \\
\hline & & & $15 \mathrm{~s}$ & $7.65 \mathrm{~s}$ & $0.97 \mathrm{~s}$ & $87.26 \%$ \\
\hline & & & $20 \mathrm{~s}$ & $10.20 \mathrm{~s}$ & $2.7 \mathrm{~s}$ & $73.5 \%$ \\
\hline \multirow{10}{*}{2000} & \multirow{10}{*}{80} & \multirow{10}{*}{40} & $1 \mathrm{~s}$ & $0.51 \mathrm{~s}$ & $0 \mathrm{~s}$ & $100 \%$ \\
\hline & & & $2 \mathrm{~s}$ & $1.02 \mathrm{~s}$ & $0 \mathrm{~s}$ & $100 \%$ \\
\hline & & & $3 \mathrm{~s}$ & $1.53 \mathrm{~s}$ & $0 \mathrm{~s}$ & $100 \%$ \\
\hline & & & $4 \mathrm{~s}$ & $2.04 \mathrm{~s}$ & $0 \mathrm{~s}$ & $100 \%$ \\
\hline & & & $5 \mathrm{~s}$ & $2.55 \mathrm{~s}$ & $0 \mathrm{~s}$ & $100 \%$ \\
\hline & & & $7 \mathrm{~s}$ & $3.57 \mathrm{~s}$ & $0 \mathrm{~s}$ & $100 \%$ \\
\hline & & & $10 \mathrm{~s}$ & $5.10 \mathrm{~s}$ & $0.13 \mathrm{~s}$ & $97.44 \%$ \\
\hline & & & $12 \mathrm{~s}$ & $6.13 \mathrm{~s}$ & $0.39 \mathrm{~s}$ & $93.53 \%$ \\
\hline & & & $15 \mathrm{~s}$ & $7.65 \mathrm{~s}$ & $1.17 \mathrm{~s}$ & $84.56 \%$ \\
\hline & & & $20 \mathrm{~s}$ & $10.20 \mathrm{~s}$ & $3.04 \mathrm{~s}$ & $70.18 \%$ \\
\hline \multirow{10}{*}{2000} & \multirow{10}{*}{120} & \multirow{10}{*}{35} & $1 \mathrm{~s}$ & $0.51 \mathrm{~s}$ & $0 \mathrm{~s}$ & $100 \%$ \\
\hline & & & $2 \mathrm{~s}$ & $1.02 \mathrm{~s}$ & $0 \mathrm{~s}$ & $100 \%$ \\
\hline & & & $3 \mathrm{~s}$ & $1.53 \mathrm{~s}$ & $0 \mathrm{~s}$ & $100 \%$ \\
\hline & & & $4 \mathrm{~s}$ & $2.04 \mathrm{~s}$ & $0.02 \mathrm{~s}$ & $98.76 \%$ \\
\hline & & & $5 \mathrm{~s}$ & $2.55 \mathrm{~s}$ & $0.04 \mathrm{~s}$ & $98.43 \%$ \\
\hline & & & $7 \mathrm{~s}$ & $3.57 \mathrm{~s}$ & $0.16 \mathrm{~s}$ & $95.28 \%$ \\
\hline & & & $10 \mathrm{~s}$ & $5.10 \mathrm{~s}$ & $0.93 \mathrm{~s}$ & $81.60 \%$ \\
\hline & & & $12 \mathrm{~s}$ & $6.13 \mathrm{~s}$ & $1.62 \mathrm{~s}$ & $73.41 \%$ \\
\hline & & & $15 \mathrm{~s}$ & $7.65 \mathrm{~s}$ & $2.92 \mathrm{~s}$ & $61.83 \%$ \\
\hline & & & $20 \mathrm{~s}$ & $10.20 \mathrm{~s}$ & $4.87 \mathrm{~s}$ & $52.24 \%$ \\
\hline
\end{tabular}

TABLE III

Handover Delay Comparison. City Beltway Scenario $(R=225 \mathrm{~m})$

\begin{tabular}{|c|c|c|c|c|c|c|}
\hline$R T T($ veh,$H A)$ & $T_{R A}$ & $\bar{T}_{\text {conf }}$ & $\bar{T}_{\text {conf }}^{\text {OH }}$ & $\bar{T}_{h o}$ & $\bar{T}_{h o}^{O H}$ & Saving \\
\hline \multirow{5}{*}{$5.37 \mathrm{~ms}$} & $1 \mathrm{~s}$ & $0.51 \mathrm{~s}$ & $0.00 \mathrm{~s}$ & $0.52 \mathrm{~s}$ & $0.02 \mathrm{~s}$ & $98.96 \%$ \\
& $4 \mathrm{~s}$ & $2.04 \mathrm{~s}$ & $0.00 \mathrm{~s}$ & $2.05 \mathrm{~s}$ & $0.05 \mathrm{~s}$ & $99.73 \%$ \\
& $10 \mathrm{~s}$ & $5.10 \mathrm{~s}$ & $0.06 \mathrm{~s}$ & $5.11 \mathrm{~s}$ & $0.07 \mathrm{~s}$ & $98.69 \%$ \\
& $20 \mathrm{~s}$ & $10.20 \mathrm{~s}$ & $2.70 \mathrm{~s}$ & $10.21 \mathrm{~s}$ & $2.71 \mathrm{~s}$ & $73.48 \%$ \\
\hline \multirow{5}{*}{$18.32 \mathrm{~ms}$} & $1 \mathrm{~s}$ & $0.51 \mathrm{~s}$ & $0.00 \mathrm{~s}$ & $0.53 \mathrm{~s}$ & $0.02 \mathrm{~s}$ & $96.53 \%$ \\
& $4 \mathrm{~s}$ & $2.04 \mathrm{~s}$ & $0.00 \mathrm{~s}$ & $2.06 \mathrm{~s}$ & $0.02 \mathrm{~s}$ & $99.11 \%$ \\
& $10 \mathrm{~s}$ & $5.10 \mathrm{~s}$ & $0.06 \mathrm{~s}$ & $5.12 \mathrm{~s}$ & $0.08 \mathrm{~s}$ & $98.44 \%$ \\
& $20 \mathrm{~s}$ & $10.20 \mathrm{~s}$ & $2.70 \mathrm{~s}$ & $10.22 \mathrm{~s}$ & $2.72 \mathrm{~s}$ & $73.39 \%$ \\
\hline & $1 \mathrm{~s}$ & $0.51 \mathrm{~s}$ & $0.00 \mathrm{~s}$ & $0.65 \mathrm{~s}$ & $0.14 \mathrm{~s}$ & $78.62 \%$ \\
& $4 \mathrm{~s}$ & $2.04 \mathrm{~s}$ & $0.00 \mathrm{~s}$ & $2.18 \mathrm{~s}$ & $0.14 \mathrm{~s}$ & $93.63 \%$ \\
& $10 \mathrm{~s}$ & $5.10 \mathrm{~s}$ & $0.06 \mathrm{~s}$ & $5.24 \mathrm{~s}$ & $0.20 \mathrm{~s}$ & $96.18 \%$ \\
& $20 \mathrm{~s}$ & $10.20 \mathrm{~s}$ & $2.70 \mathrm{~s}$ & $10.34 \mathrm{~s}$ & $2.84 \mathrm{~s}$ & $72.53 \%$ \\
\hline
\end{tabular}

\section{EXPERIMENTAL RESUlts}

To be able to conduct real experiments that allowed us to evaluate the performance of our overhearing-assisted optimization for GeoSAC, we developed a prototype of both GeoSAC and our proposed optimization. The prototype was implemented for Linux in user space. One of the main challenges was to deploy an experimental setup that allowed us to emulate a portion of a highway populated with vehicles. We used the Linksys WRT54GL v1.1 router as hardware for the vehicular communication box. This is a small and very popular home and office broadband router equipped with a $200-\mathrm{MHz}$ processor, an IEEE $802.11 \mathrm{~b} / \mathrm{g}$ WLAN interface, and an IEEE 802.3 Ethernet interface connected to a virtual LAN capable five-port switch. The firmware of the router can be replaced with an open-source Linux-based firmware. We installed the
OpenWRT ${ }^{12}$ Backfire 10.03.1-rc4 distribution with a Linux-2.4 kernel in the routers. This firmware gave us more flexibility in the use and configuration of the routers than the original firmware. A wired interface of each of the routers is used to perform several control and management plane operations, such as the global synchronization of the routers, the remote execution of tests, and the retrieval of the results for offline processing. A total of 40 routers were used for the tests. Additionally, we used a laptop as RSU and controlling node to monitor and manage all the routers of our deployment through the wired interfaces. The nodes calculated their geographical position with the help of timeframe messages broadcast by the controller node on the

\footnotetext{
${ }^{12} \mathrm{http} / / /$ www.openwrt.org/
} 


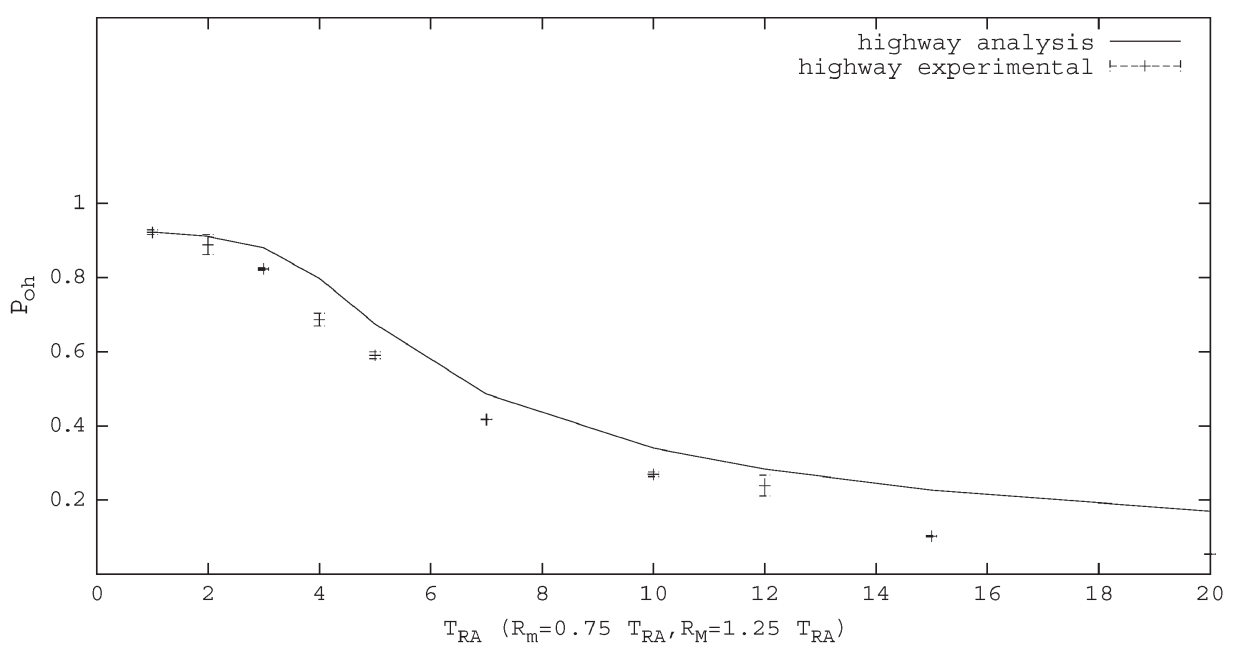

(a)

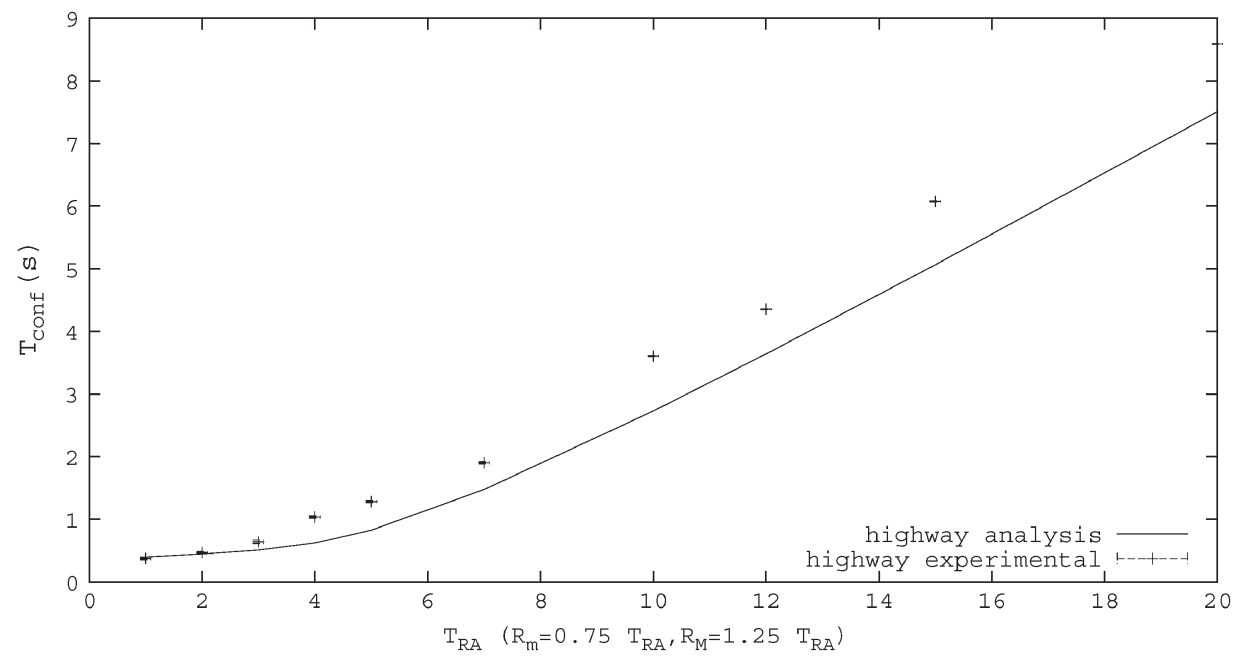

(b)

Fig. 12. Analysis and experimental results, highway scenario, $D_{\mathrm{RSU}}=1000 \mathrm{~m}, R=150 \mathrm{~m}$. (a) Overhearing probability. (b) IP address configuration time.

wired management network. The protocol was implemented as explained in Section III-A.

The testbed was physically deployed in a laboratory of our computer science building. Due to the fact that all the routers were within wireless radio connectivity range, iptables software was used to selectively filter the packets that each router received and, this way, be able to emulate any given physical topology. The controlling node computed a random topology (initial position and speed of each vehicle) at the beginning of each run and then remotely configured each Linksys router using iptables so that the routers emulated the topology and movement of the nodes during the test. On each run, we collected the results (IP address configuration time and overhearing success) of all the vehicles that could potentially be configured with an overhearing success (i.e., those farther than $R$ meters from the area border, the rest are used to populate the highway segment between the RSU and the unconfigured nodes) and processed them at the controlling node. Different inter-RA values were configured, and each test was composed of 300 iterations. We evaluated the highway scenario with $D_{\mathrm{RSU}}=1000 \mathrm{~m}$ and $R=150 \mathrm{~m}$.
The obtained results (displayed in Fig. 12) show that the experimental performance is quite close to that predicted by our theoretical analysis. The difference between the experimental and theoretical results can be caused by the fact that the wireless media is more crowded in our laboratory environment than in a real highway because all the nodes are placed together in the same room and the presence of other interfering external wireless networks. Nevertheless, it is worth highlighting that our model matches quite reasonably the experimental results obtained from our testbed, and this supports the feasibility of the implementation of our overhearing-assisted optimization in a real prototype.

\section{CONCLUSION}

This paper has presented an overhearing-assisted optimization for GeoSAC consisting of an address autoconfiguration mechanism for vehicular networks. The optimization is based on vehicles overhearing RAs generated at neighboring geographical areas and allowing the vehicles to precompute valid IP addresses to be used at those areas in the event that they 
enter one of them. This optimization does not require changes to the operation protocol of the original GeoSAC, and it is fully compatible with nodes compliant to GeoSAC.

This paper provides an analytical expression for the probability of our optimization being used effectively in different realistic scenarios that consider values for vehicular density, coverage radius of the wireless technology, and distance between attachment points deployed by the road infrastructure. We have also derived an expression for the IP address autoconfiguration time when our optimization is enabled and have compared it with the time achieved by GeoSAC under optimal conditions. The analytical work has been validated by means of extensive simulation, including real wireless models and experiments incorporating vehicular traces from Spain. Additionally, we have conducted an experimental evaluation using a real implementation of our solution in a testbed composed of 40 nodes.

Due to the properties of our optimization, we have shown in this paper that it can be used not only to decrease the IP address configuration time but to reduce the network signaling load (in terms of unsolicited RAs) required to achieve a certain target configuration time as well. Finally, we have also analyzed the benefits that our optimization provides when the vehicle uses an IP mobility protocol to enable transparent connectivity to the Internet despite changes in geographical areas.

\section{ACKNOWLEDGMENT}

The authors would like to thank G. de Veciana, P. Serrano, and J. Barceló for their very useful revisions of previous versions of this paper and would like to acknowledge the Spanish Directorate General of Traffic for kindly proving us with the empirical traces used in this work. The authors would also like thank the anonymous reviewers for their valuable comments.

\section{REFERENCES}

[1] V. Bychkovsky, B. Hull, A. Miu, H. Balakrishnan, and S. Madden, "A measurement study of vehicular Internet access using in situ Wi-Fi networks," in Proc. 12th ACM MobiCom, San Francisco, CA, 2006, pp. 50-61.

[2] P. Belanovic, D. Valerio, A. Paier, T. Zemen, F. Ricciato, and C. Mecklenbräuker, "On wireless links for vehicle-to-infrastructure communications,” IEEE Trans. Veh. Technol., vol. 59, no. 1, pp. 269-282, Jan. 2010.

[3] J. Marquez-Barja, C. Calafate, J.-C. Cano, and P. Manzoni, "Multi-layer performance evaluation of a content delivery framework for urban vehicular networks," in Proc. IEEE ICC Workshops, 2010, pp. 1-5.

[4] K. Abrougui, A. Boukerche, and R. Pazzi, "Location-aided gateway advertisement and discovery protocol for VANets," IEEE Trans. Veh. Technol., vol. 59, no. 8, pp. 3843-3858, Oct. 2010.

[5] R. Baldessari, A. Festag, W. Zhang, and L. Le, "A MANET-centric solution for the application of NEMO in VANET using geographic routing,' in Proc. TridentCom, Innsbruck, Austria, Mar. 2008, pp. 1-7.

[6] J. Choi, Y. Khaled, M. Tsukada, and T. Ernst, "IPv6 support for VANET with geographical routing," in Proc. 8th Int. Conf. ITST, Oct. 2008, pp. 222-227.

[7] R. Baldessari, C. J. Bernardos, and M. Calderon, "GeoSAC-Scalable address autoconfiguration for VANET using geographic networking concepts," in Proc. PIMRC, Cannes, France, Sep. 2008, pp. 1-7.

[8] S. Thomson, T. Narten, and T. Jinmei, IPv6 Stateless Address Autoconfiguration, RFC 4862, Sep. 2007.

[9] T. Narten, E. Nordmark, W. Simpson, and H. Soliman, Neighbor Discovery for IP ver. 6 (IPv6), RFC 4861, Sep. 2007.

[10] R. Droms, J. Bound, B. Volz, T. Lemon, C. Perkins, and M. Carney, Dynamic Host Configuration Protocol for IPv6 (DHCPv6), RFC 3315, Jul. 2003.
[11] D. Kim, H.-J. Jeong, C. K. Toh, and S. Oh, "Passive duplicate addressdetection schemes for on-demand routing protocols in mobile ad hoc networks," IEEE Trans. Veh. Technol., vol. 58, no. 7, pp. 3558-3568, Sep. 2009.

[12] M. Fazio, S. Das, C. Palazzi, and M. Gerla, "Vehicular address configuration," in Proc. 1st IEEE Workshop AutoNet, GLOBECOM, San Francisco, CA, 2006.

[13] B. K. Mohandas and R. Liscano, "IP address configuration in VANET using centralized DHCP," in Proc. 33rd IEEE Local Comput. Netw. Conf., 2008, pp. 608-613.

[14] Intelligent Transport Systems (ITS); Vehicular Communications; GeoNetworking; Part 3: Network Architecture, ETSI TS 102 636-3 V1.1.1, Mar. 2010.

[15] V. Devarapalli, R. Wakikawa, A. Petrescu, and P. Thubert, Network Mobility (NEMO) Basic Support Protocol, RFC 3963, Jan. 2005.

[16] Intelligent Transport Systems (ITS); Vehicular Communications; Part 4: Geographical Addressing and Forwarding for Point-to-Point and Point-toMultipoint Communications; Sub-Part 1: Media-Independent Functionality, ETSI TS 102 636-4-1 (work in progress), Jun. 2010.

[17] Intelligent Transport Systems (ITS); Vehicular Communications, Part 6: Internet Integration; Sub-Part 1: Transmission of IPv6 Packets Over GeoNetworking Protocols, ETSI TS 102 636-6-1 (work in progress), Jun. 2010.

[18] J. Ott and D. Kutscher, "The drive-thru architecture: WLAN-based Internet access on the road," in Proc. VTC-Spring, May 2004, pp. 2615-2622.

[19] D. Johnson, C. Perkins, and J. Arkko, Mobility Support in IPv6, RFC 3775, Jun. 2004.

[20] C. J. Bernardos, I. Soto, M. Calderon, F. Boavida, and A. Azcorra, "VARON: Vehicular ad-hoc route optimisation for NEMO," Comput. Commun., vol. 30, no. 8, pp. 1765-1784, Jun. 2007.

[21] Q. Mussabbir, W. Yao, Z. Niu, and X. Fu, "Optimized FMIPv6 using IEEE 802.21 MIH services in vehicular networks," IEEE Trans. Veh. Technol., vol. 56, no. 6, pp. 3397-3407, Nov. 2007.

[22] N. Wisitpongphan, F. Bai, P. Mudalige, V. Sadekar, and O. Tonguz, "Routing in sparse vehicular ad hoc wireless networks," IEEE J. Sel. Areas Commun., vol. 25, no. 8, pp. 1538-1555, Oct. 2007.

[23] S. Yousefi, E. Altaian, and R. El-Azouzi, "Study of connectivity in vehicular ad hoc networks," in Proc. 5th Int. Symp. Model. Optim. Mobile, Ad Hoc Wireless Netw. Workshops, WiOpt, Apr. 2007, pp. 1-6.

[24] H. Reijmers and R. Prasad, "The influence of vehicle distribution models on packet success probability on a three lane motorway," in Proc. 48th IEEE VTC, 1998, vol. 3, pp. 1785-1789.

[25] L. Bain and D. Weeks, "A note on the truncated exponential distribution," Ann. Math. Statist., vol. 35, no. 3, pp. 1366-1367, Sep. 1964.

[26] A. Köpke, M. Swigulski, K. Wessel, D. Willkomm, P. T. K. Haneveld, T. E. V. Parker, O. W. Visser, H. S. Lichte, and S. Valentin, "Simulating wireless and mobile networks in OMNeT++ the Mixim vision," in Proc. 1st Int. Conf. Simul. Tools Techn. Commun., Netw. Syst. Workshops, Simutools, 2008, pp. 1-8.

[27] J. K. Cavers, Mobile Channel Characteristics. Norwell, MA: Kluwer, 2000.

[28] M. K. Simon and M.-S. Alouini, "Digital communications over fading channels (M.K. Simon and M.S. Alouini; 2005) [book review]," IEEE Trans. Inf. Theory, vol. 54, no. 7, pp. 3369-3370, Jul. 2008.

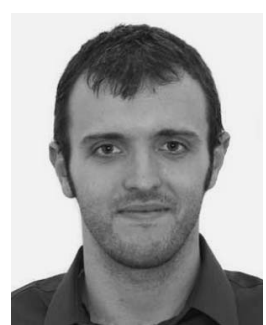

Marco Gramaglia received the B.Sc. and M.Sc. degrees in computer science in 2006 and 2008, respectively, from the Polytechnic University of Turin (Politecnico di Torino), Torino, Italy, and the M.Sc. degree in telematics in 2009 from the University Carlos III of Madrid, Leganes, Spain, where he is currently working toward the Ph.D. degree in telematics.

He is currently a Research Assistant with Institute IMDEA Networks, Leganes. 


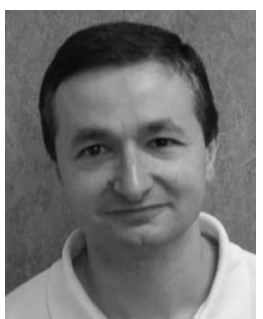

Ignacio Soto received the telecommunication engineering degree and the Ph.D. degree in telecommunications from the University of Vigo, Vigo, Spain, in 1993 and 2000, respectively.

He was a Research and Teaching Assistant in telematics engineering with the University of Valladolid, Valladolid, Spain, from 1993 to 1999. In 1999, he was with the University Carlos III of Madrid, Leganes, Spain, where he was an Associate Professor until 2010. Since 2010, he has been an Associate Professor with the Universidad Politécnica de Madrid, Madrid, Spain. His research activities focus on mobility support in packet networks, heterogeneous wireless access networks, and automatic network management and operation.

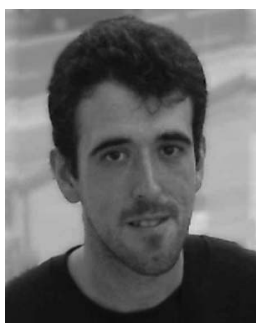

Carlos J. Bernardos received the Telecommunication Engineering degree and the Ph.D. degree in telematics from the University Carlos III of Madrid (UC3M), Leganes, Spain, in 2003 and 2006, respectively. His Ph.D. thesis focused on route optimization for mobile networks in IPv6 heterogeneous environments.

He was a Research and Teaching Assistant from 2003 to 2008 and has been an Associate Professor since 2008 with UC3M. His current work focuses on vehicular networks and Internet Protocol-based mobile communication protocols. He has published over 30 scientific papers in prestigious international journals and conferences, and he is also an active contributor to the Internet Engineering Task Force.

Dr. Bernardos served as the Technical Program Committee (TPC) Chair of the 2009 Workshop on Experimental Evaluation and Deployment Experiences on Vehicular Networks and as TPC Cochair of the mobility track of the 2011 International Conference on New Technologies, Mobility, and Security. He has also served as Guest Editor of IEEE NETWORK.

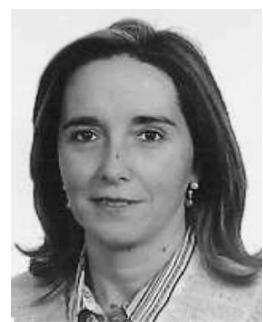

Maria Calderon received the Computer Science Engineering degree and the Ph.D. degree in computer science from the Technical University of Madrid, Madrid, Spain, in 1991 and 1996, respectively.

She is currently an Associate Professor with the Telematics Engineering Department, University Carlos III of Madrid, Leganes, Spain. She has published over 40 papers in the fields of advanced communications, reliable multicast protocols, programmable networks, and IPv6 mobility. Her current work focuses on vehicular networks and Internet Protocol-based mobile communication protocols. 